\title{
Visual Neuroscience Methods for Marmosets: Efficient Receptive Field Mapping and Head-Free Eye Tracking
}

\author{
DPatrick Jendritza, ${ }^{1,2}$ Frederike J. Klein, ${ }^{1}$ Gustavo Rohenkohl, ${ }^{1,3}$ and Pascal Fries ${ }^{1,4}$
}

https://doi.org/10.1523/ENEURO.0489-20.2021

${ }^{1}$ Ernst Strüngmann Institute (ESI) for Neuroscience in Cooperation with Max Planck Society, 60528 Frankfurt, Germany, ${ }^{2}$ International Max Planck Research School for Neural Circuits, 60438 Frankfurt, Germany, ${ }^{3}$ Department of Physiology, Institute of Biosciences, University of São Paulo, 05508-000 São Paulo, Brazil, and ${ }^{4}$ Donders Institute for Brain, Cognition and Behaviour, Radboud University Nijmegen, 6525 EN Nijmegen, The Netherlands

\begin{abstract}
The marmoset has emerged as a promising primate model system, in particular for visual neuroscience. Many common experimental paradigms rely on head fixation and an extended period of eye fixation during the presentation of salient visual stimuli. Both of these behavioral requirements can be challenging for marmosets. Here, we present two methodological developments, each addressing one of these difficulties. First, we show that it is possible to use a standard eye-tracking system without head fixation to assess visual behavior in the marmoset. Eye-tracking quality from head-free animals is sufficient to obtain precise psychometric functions from a visual acuity task. Second, we introduce a novel method for efficient receptive field (RF) mapping that does not rely on moving stimuli but uses fast flashing annuli and wedges. We present data recorded during head-fixation in areas V1 and V6 and show that RF locations are readily obtained within a short period of recording time. Thus, the methodological advancements presented in this work will contribute to establish the marmoset as a valuable model in neuroscience.
\end{abstract}

Key words: eye tracking; head-free; marmoset; receptive field mapping

\section{Significance Statement}

The marmoset monkey is becoming an increasingly relevant model for biological and medical research. Here, we present two methodological advancements for visual neuroscience that are adapted to the marmoset. First, we present a head-free eye-tracking protocol that is sufficiently accurate for a large variety of visual experiments. Second, we introduce an efficient technique for mapping visual receptive fields (RFs) and apply it to map RFs of neurons from the visual cortex of head-fixed marmosets. The concepts presented in this work can be easily transferred to other species. Together, this will promote diversification of the animal model landscape and solidify the contribution of marmoset research.

\section{Introduction}

The common marmoset (Callithrix jacchus) has recently gained enormous popularity as an emerging model for neuroscience (Servick, 2018). Marmosets combine

\footnotetext{
Received November 15, 2020; accepted March 25, 2021; First published April 16, 2021.

P.F. is beneficiary of a license contract on thin-film electrodes with Blackrock Microsystems LLC, member of the Scientific Technical Advisory Board of CorTec $\mathrm{GmbH}$, and managing director of Brain Science $\mathrm{GmbH}$. All other authors declare no competing financial interests.
}

several advantages as a model animal: they are small in size, have fast reproduction cycles, and their rich behavioral repertoire makes them ideal to study a variety of complex and social behaviors (Stevenson and Poole, 1976; Koski and Burkart, 2015; Miller et al., 2016).

Author contributions: P.J., F.J.K., and P.F. designed research; P.J. and F.J.K. performed research; P.J. and G.R. analyzed data; P.J., G.R., and P.F. wrote the paper. 
Recently, there have been successes in creating transgenic marmosets as disease models (Sasaki et al., 2009; Tomioka et al., 2017a,b; Sato et al., 2020) and in the use of genetically encoded calcium indicators (Park et al., 2016). Because of the relatively high reproduction rates and the fact that marmosets often give birth to twins, it is likely that the marmoset will become a viable transgenic primate model (Shen, 2013; Kishi et al., 2014; Mitchell and Leopold, 2015).

The marmoset is of particular interest for the field of visual neuroscience (Solomon and Rosa, 2014; Mitchell and Leopold, 2015). Marmosets rely strongly on their sense of sight and therefore have a highly developed visual system. This is reflected in the fact that a large fraction of their neocortex is dedicated to visual processing (Rosa et al., 2009) and by the occurrence of brain networks for the processing of complex visual objects, e.g., faces (Hung et al., 2015). Because of the lissencephalic nature of the marmoset cortex (Heide et al., 2020), many visual areas are exposed on the surface of the brain, making them directly accessible for neuronal recording and imaging techniques. This enables the investigation of high-level brain areas that do not have clear homologues in the rodent and are difficult to reach in larger primates.

Previous neurophysiological studies have provided substantial groundwork on various aspects of the visual system of the marmoset (for review, see Solomon and Rosa, 2014). However, the majority of visual experiments in marmosets have been performed under anesthesia, thus making it impossible to identify the neuronal circuits underlying visual behavior. Therefore, it is crucial to develop methods for monitoring and manipulating neuronal activity in the awake animal.

Studies in the awake marmoset have predominantly been performed under head fixation. This has been used for various recording and stimulation approaches, like electrocorticographic (Hung et al., 2015), extracellular microelectrode (Remington et al., 2012; Johnston et al., 2018), and intracellular (Gao et al., 2016; Gao and Wang, 2019) neuronal recordings, microstimulation (Selvanayagam et al., 2019), fMRI (Belcher et al., 2013; Liu et al., 2013; Hung et al., 2015; Schaeffer et al., 2019), as well as calcium imaging (Yamada et al., 2004; Mehta et al., 2019) and optogenetics (Macdougall et al., 2016; Ebina et al., 2019). Psychophysical

P.F. was supported by German Research Foundation (DFG) Grants SPP 1665 FR2557/1-1, FOR 1847 FR2557/2-1, FR2557/5-1-CORNET, FR2557/6-1NeuroTMR, and FR2557/7-1 DualStreams; European Union Grants HEALTH-F22008-200728-BrainSynch, FP7-604102-HBP, and FP7-600730-Magnetrodes; a European Young Investigator Award; the National Institutes of Health Grant 1U54MH091657-WU-Minn-Consortium-HCP; and LOEWE (NeFF). G.R. is supported by Fundação de Amparo à Pesquisa do Estado de São Paulo (FAPESP) Grants 2017/10429-5 and 2018/16635-9.

Acknowledgements: We thank Marianne Hartmann at the ESI for her continuous support in training the animals.

Correspondence should be addressed to Patrick Jendritza at patrick.jendritza@esi-frankfurt.de.

https://doi.org/10.1523/ENEURO.0489-20.2021

Copyright @ 2021 Jendritza et al.

This is an open-access article distributed under the terms of the Creative Commons Attribution 4.0 International license, which permits unrestricted use, distribution and reproduction in any medium provided that the original work is properly attributed. studies of marmoset vision have, to our knowledge, exclusively used head-fixed conditions, allowing precise eye tracking (Mitchell et al., 2014, 2015; Nummela et al., 2017). However, head-fixation can conflict with the execution of normal behaviors (Populin, 2006; Pandey et al., 2020), exposing the necessity for more naturalistic and less constrained paradigms (Krakauer et al., 2017; Sonkusare et al., 2019). Such paradigms have recently been successfully used to study the motor system (Kondo et al., 2018; Mundinano et al., 2018; Umeda et al., 2019), spatial navigation (Courellis et al., 2019), and the auditory system (Eliades and Wang, 2008; Roy and Wang, 2012) of the marmoset. Similar to the experimental setup, the design of stimuli can also be guided by the intrinsic behavior of an animal to gain understanding about brain function (Knöll et al., 2018).

The overall goal of this work was to adapt methods for visual neuroscience, such that they are more suitable for the behavioral requirements of the marmoset. We applied this idea to two key methods: eye tracking and receptive field (RF) mapping. First, we remove the necessity for head fixation in visual psychophysics experiments and show that eye-tracking quality from head-free animals is sufficient to measure precise psychometric functions. Second, we present a novel adaptation of visual stimuli for efficient mapping of RFs and provide neuronal data from areas $\mathrm{V} 1$ and $\mathrm{V} 6$ recorded under head-fixation.

\section{Materials and Methods}

All animal experiments were approved by the responsible government office (Regierungspräsidium Darmstadt) in accordance with the German law for the protection of animals and the European Union's Directive 2010/63/EU.

\section{Animals}

Five male adult common marmosets (C. jacchus) were included in this study. The decision to use male animals was due to availability only and was not part of the experimental design. Data from one other animal was excluded because the number of collected trials in the visual acuity task was much lower than in the other animals (excluded animal: $n=1637$ hits, vs. Monkey D: $n=4877$ hits, Monkey U: $n=3918$ hits, Monkey E: $n=4838$ hits, Monkey X: $n=2951$ hits). Animals were typically housed in groups of two or three. The housing area was kept at a temperature of $23-28^{\circ} \mathrm{C}$ and at a humidity level of $40-70 \%$. The dark/ light cycle was $12 \mathrm{~h} / 12 \mathrm{~h}$, switching at 6 A.M./6 P.M.

\section{Food schedule and reward}

Animals were fed in their home cages with marmoset pellets, nuts, fresh fruits and vegetables. Animals were on a mild food schedule and had ad libitum access to water. Typically, food was removed from the home cage after 17:00, and animals went into training/recording sessions the following day between 10 A.M. and 2 P.M. No large changes in body weight in relation to the training were observed. The reward during the tasks was a viscous solution of gum arabic (gum arabic powder, Willy Benecke) applied through a syringe pump (AL-1000HP, WPI) that was controlled by a custom Arduino-based circuit. The amount of reward was typically between 0.05 and $0.09 \mathrm{ml}$ per trial, and manually adjusted according to the performance of the animal. 
A

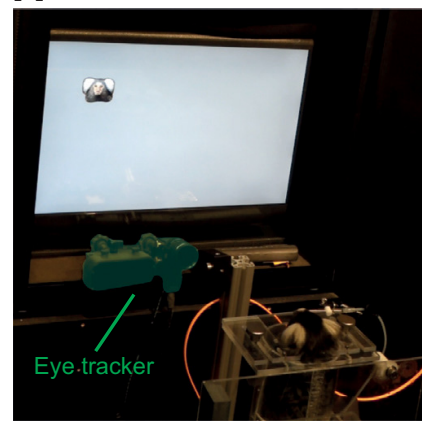

B

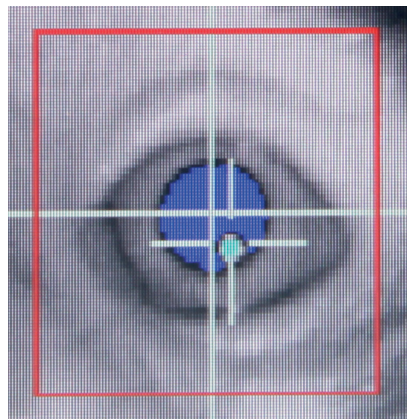

Figure 1. Head-free eye-tracking setup. A, Experimental setup with a marmoset performing a simple face detection task. Eyetracking camera and IR-light source are highlighted in green. $\boldsymbol{B}$, Online view of the eye-tracking software. Pupil and corneal reflex are being tracked.

\section{Behavioral training}

Naive animals were first slowly acclimatized to be transported inside a dedicated transport box from the housing area to the laboratory setup. In the setup, they were allowed to enter the primate chair through a tunnel that connected the transport box with the chair. The position of the chair remained fixed across sessions, and the opening for the head was minimized ( $36 \mathrm{~mm}$ wide and $40 \mathrm{~mm}$ long), which assured that the head remained within a small region across sessions. As soon as the animals felt comfortable to stick out their head from the chair, they were rewarded manually through a lick spout that was placed in front of the animal's mouth. The lick spout position was adjusted per session with regard to horizontal distance to the animal's mouth to enable easy licking, yet the lateral and vertical position remained fixed across sessions. As the animals developed a stereotyped licking routine, this further contributed to constant head positioning during eye tracking. After this initial training, the setup was configured to automatically reward the animal whenever an eye signal could be detected. This led to the animal being conditioned to face forward and to look directly at the monitor. At this stage, animals were ready to be presented with visual stimuli that could be used to perform the initial eye calibration. For this, we showed small marmoset faces at defined positions on the monitor (Fig. 1A).

\section{Stimulus presentation}

Stimulus presentation was controlled by the customdeveloped ARCADE toolbox (https://github.com/esineuroscience/ARCADE), based on MATLAB (MathWorks) and $\mathrm{C}++$. Stimuli were displayed on a TFT monitor (SyncMaster 2233RZ, Samsung) at a refresh rate of $120 \mathrm{~Hz}$. The monitor was gamma corrected and placed at a distance of $45 \mathrm{~cm}$ in front of the animal, resulting in $27.854 \mathrm{pixel} /$ degree of visual angle. Animals performed the task in a dimly lit recording booth. A photodiode was placed in the top left corner of the monitor to determine exact stimulus-onset times.

\section{Eye tracking}

The left eye of the animals was tracked at $1 \mathrm{kHz}$ sampling rate with a commercial eye-tracking system (Eyelink
1000, SR Research). Corneal reflex and pupil were tracked under external illumination with infrared light. A $25 \mathrm{~mm} / \mathrm{F} 1.4$ lens was used at a distance of $28 \mathrm{~cm}$ to the animal's eye. This resulted in a relatively large field of view, allowing for eye tracking despite head movement.

\section{Calibration and analysis of eye data}

An initial coarse calibration via the Eyelink software was performed before using the system to collect data for offline calibration. For this, we used large salient stimuli (e.g., faces) presented at the default Eyelink calibration points (nine-point calibration). We manually accepted the position of the gaze to the target location with a key press. This initial coarse calibration was then used for each animal to perform the actual calibration task with large tracking windows (3-4 degrees). During the calibration task, a fixation point was shown that consisted of two overlaid Gaussians (one displayed in the background and colored green with a size of 0.15 degree SD, the other one black with a size of 0.05 degree SD). Animals were required to fixate the fixation point for $150-300 \mathrm{~ms}$, at which time a small black Gaussian stimulus ( 0.08 degree SD) was presented at one of nine possible calibration positions: $0 / 0,-300 /-150$, $-300 / 0,-300 / 150,0 / 150,300 / 150,300 / 0,300 /-150,0 /-150$ pixels (300 pixels $\approx 10.77$ degree), referenced from the center of the monitor (Fig. 2A). After a correct saccade to the target (reaching a window of 3-4 degrees around the target within $50-800 \mathrm{~ms}$ after target onset) and $100 \mathrm{~ms}$ of fixation on the target, a picture of a marmoset face was displayed, and the animal was rewarded.

The uncalibrated eye data were plotted as shown in Figure $2 A$, and the positions with highest density were manually selected. The extracted coordinates from the selected eye positions and the known calibration points were then used to fit a third-order 2D polynomial function (fitgeotrans function in MATLAB) to generate a template calibration for each animal.

For the estimation of accuracy (offset) and precision (sigma), 153 sessions from four marmosets were analyzed (Monkey D: $n=38$ sessions, Monkey U: $n=37$ sessions, Monkey E: $n=40$ sessions, Monkey X: $n=38$ sessions). Six sessions were excluded, because eye data were lost due to storage issues. For every session, eye data were binned with a bin size of 0.05 degree. The $x$ and $y$ components of the central 2.5 degrees were averaged and fitted with a 1D Gaussian function, separately per animal and session. The mean of the Gaussian fit corresponds to the $\mathrm{X}$ - and Y-offset from zero. The Euclidean distance from zero to the $\mathrm{X}$ - and $\mathrm{Y}$-offset values from the fits was taken as the absolute offset. The Euclidean distance from zero to the $X$ - and $Y$ - sigma-values from the fits was taken as the absolute sigma value.

\section{Visual acuity task}

Animals were required to fixate a central fixation point for $350-800 \mathrm{~ms}$. After this period, a small Gabor stimulus ( $50 \%$ contrast, 0.3 degree SD in size, random orientation) was presented at one of eight possible equi-eccentric locations at a distance of 300 pixels $(\approx 10.77$ degrees) from the center. No corrective changes to the stimulus position were made. Spatial frequency values varied across trials 
A

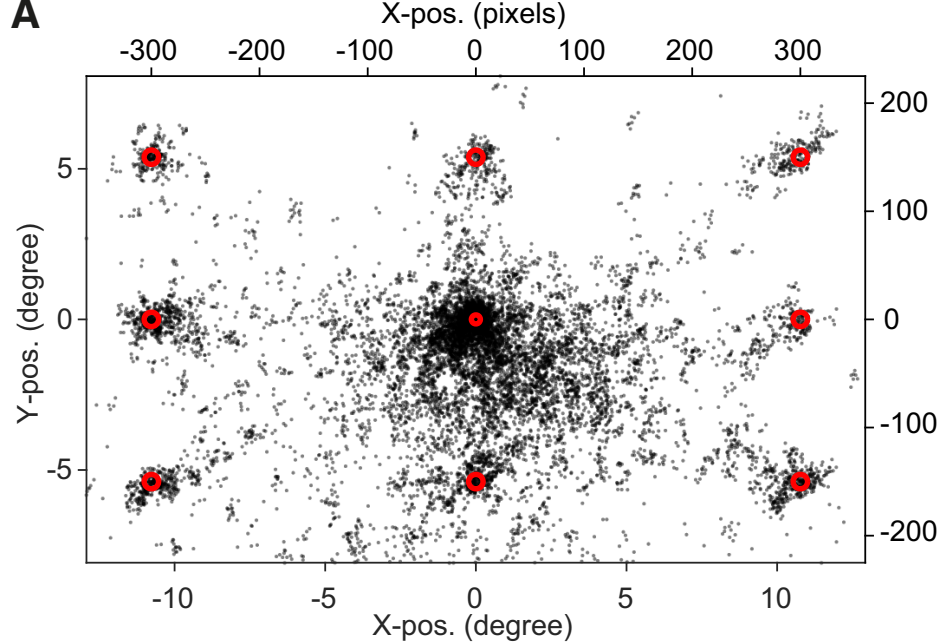

$\mathrm{X}$-pos. (pixels)

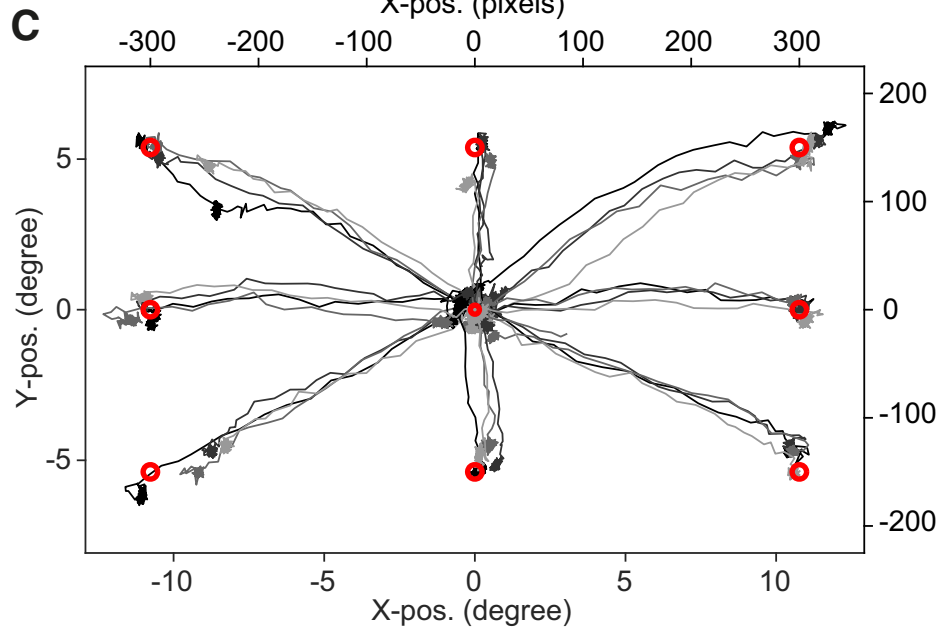

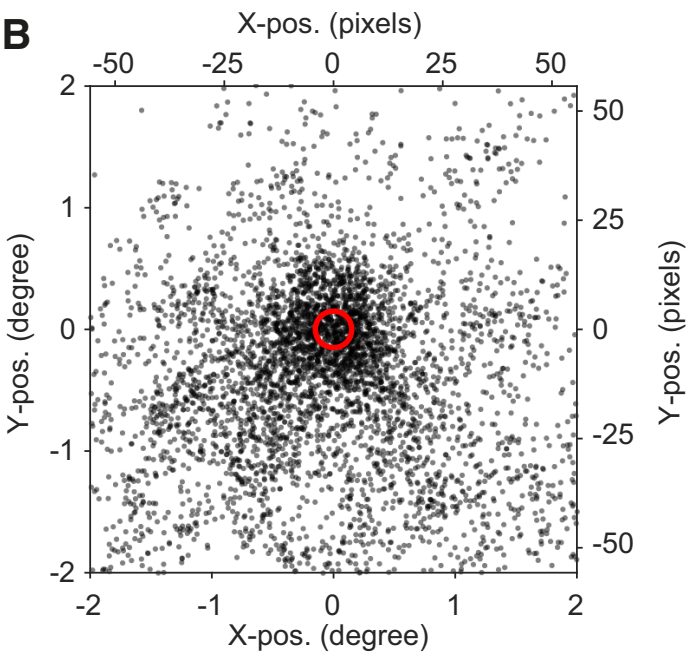

D

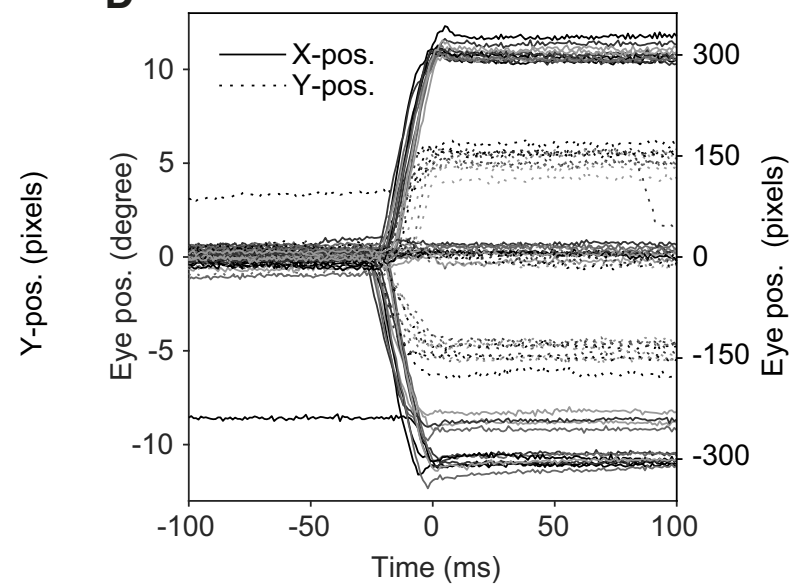

Figure 2. Offline calibration approach. $\boldsymbol{A}$, Scatter plot of eye positions from an example calibration session. Every 25 th sample is shown with $50 \%$ transparency for better visibility. Red circles indicate six times the SD of the Gaussians used as visual stimuli or fixation point in the task. $\boldsymbol{B}$, Same data as in $\boldsymbol{A}$ but only showing the central 2 degree. $\boldsymbol{C}$, Saccade traces of four example trials per target position from the same data. $\boldsymbol{D}$, Same data as in $\mathbf{C}$ but plotted as a function of time for X-and Y-positions separately. Data are aligned to the moment when gaze position entered the correct target window. Note that the example trials (first four trials per condition from an example session) include one trial with a double saccade and three trials with undershooting saccades.

and could assume the following values: $1.5,2.75,4.0$, $5.25,6.5,7.75,9.0,10.25,11.5$ cycles/degree, with the probability of the lowest spatial frequency condition being twice as high as any of the other conditions. Trials were categorized as hits if the animal made a saccade to this stimulus within $500 \mathrm{~ms}$. Responses that were faster than $50 \mathrm{~ms}$ were categorized as early responses and were not rewarded. After a correct saccade to the target, a picture of a marmoset face was displayed and the animal was rewarded. The amount of reward was typically between 0.05 and $0.09 \mathrm{ml}$ per trial, and manually adjusted according to the performance of the animal. Animals were rewarded with a small reward $(0.0025 \mathrm{ml})$ when they missed the target but maintained fixation until the end of the trial.

\section{Passive fixation task}

Reference data for eye-tracking quality from head-fixed animals were obtained from a passive fixation task.
Animals were required to fixate a central fixation point for $100-140 \mathrm{~ms}$. After this period, a grating stimulus was presented for $500 \mathrm{~ms}$, and the animal was required to maintain fixation throughout the trial to obtain reward. Calibration was performed as in the head-free experiments and was kept identical across sessions.

\section{Psychometric analysis}

We calculated hit rates and mean reaction times (RTs) for all spatial frequency conditions and fitted the following four-parameter logistic model to the data (Cardillo, 2012):

$$
f(x)=\gamma+\frac{\lambda-\gamma}{1+\left(\frac{x}{\alpha}\right)^{\beta}},
$$

where $\gamma$ is the lower asymptote of the logistic function (fixed at chance performance of $12.5 \%$, for the hit rate 
data, and restricted to be between 50 and $500 \mathrm{~ms}$ for the RT data), and $\lambda$ is the upper asymptote (restricted to be between 12.5-100\% for the hit rate data, and between 50 and $500 \mathrm{~ms}$ for the RT data). The $\alpha$ parameter corresponds to the inflection point and gives the spatial frequency value at which the hit rate, or mean $\mathrm{RT}$, is halfway between the lower and upper asymptote. This parameter is also called the "acuity threshold." The $\beta$ parameter determines the steepness of the curve. Confidence intervals for RTs were calculated by bootstrapping (bias corrected and accelerated percentile method with 10,000 bootstrap replications). Confidence intervals for hit rates were calculated with the Clopper-Pearson method (Clopper and Pearson, 1934).

\section{Surgical procedures}

A detailed account of all surgical procedures and recording methods will be described in a separate publication. In brief, animals were first implanted with a custom machined titanium head-post and a 3D-printed titanium chamber. In a second surgery, silicon probes were semichronically implanted in areas V1 and V6 with one microdrive per area (Nano-Drive CN-01 V1, Cambridge NeuroTech). Two 32-channel shanks with $250-\mu \mathrm{m}$ spacing were implanted in V1, and four 32-channel shanks in V6 (H2 probe, Cambridge NeuroTech). For experiments not described here, a viral vector (AAV1.CamKIla. Chronos-eYFP-WPRE) was injected into area V6 just before electrode implantation. For monkey $A$, the stereotaxic coordinates from Paxinos et al. (2012) served as the anatomic guide for electrode implantation: the target coordinates for $\mathrm{V} 1$ were $8.5 \mathrm{~mm}$ caudal from the interaural line and $1.3 \mathrm{~mm}$ lateral from the midline, and the coordinates for V6 were $2.5 \mathrm{~mm}$ caudal from the interaural line and 3 $\mathrm{mm}$ lateral from the midline. For Monkey $\mathrm{D}$, we used a combination of Paxinos et al. (2012) and a CT scan of the animal's skull and chamber, to which an MRI template brain (Liu et al., 2018) was manually aligned: the target coordinates for $\mathrm{V} 1$ were $7.7 \mathrm{~mm}$ caudal from the interaural line and $1.3 \mathrm{~mm}$ lateral from the midline, and the coordinates for V6 were $2.6 \mathrm{~mm}$ caudal from the interaural line and $4.1 \mathrm{~mm}$ lateral from the midline.

Anesthesia was induced with an intramuscular injection of a mixture of alfaxalone $(8.75 \mathrm{mg} / \mathrm{kg})$ and diazepam $(0.625 \mathrm{mg} / \mathrm{kg})$. Tramadol $(1.5 \mathrm{mg} / \mathrm{kg})$ and metamizol $(80 \mathrm{mg} / \mathrm{kg})$ were injected intramuscularly for initial analgesic coverage. Subsequently, a continuous intravenous infusion was provided through the lateral tail vein to the animal. The intravenous mixture contained glucose, amino acids (Aminomix 1 Novum, Fresenius Kabi), dexamethasone $\left(0.2-0.4 \mathrm{mg} \mathrm{kg}^{-1} \mathrm{~h}^{-1}\right)$, tramadol $(0.5-1.0 \mathrm{mg}$ $\left.\mathrm{kg}^{-1} \mathrm{~h}^{-1}\right)$, and metamizol (20-40 $\left.\mathrm{mg} \mathrm{kg}^{-1} \mathrm{~h}^{-1}\right)$. The maximal infusion rate was $5 \mathrm{ml} \mathrm{kg}^{-1} \mathrm{~h}^{-1}$. After ensuring appropriate depth of anesthesia, animals were placed in a stereotaxic frame. Animals were breathing spontaneously throughout the surgery via a custom face mask that applied isoflurane $\left(0.5-2 \%\right.$ in $\left.100 \% \mathrm{O}_{2}\right)$. Heart rate, respiration rate and body temperature were constantly monitored (Model 1030 Monitoring \& Gating System, SAII).

\section{Acquisition and processing of neuronal data}

Neuronal signals were recorded through active, unity gain head stages (ZC32, Tucker Davis Technologies), digitized at $24,414.0625 \mathrm{~Hz}$ (PZ2 preamplifier, Tucker Davis Technologies) and re-sampled offline to $25 \mathrm{kHz}$. Sample-by-sample re-referencing was applied by calculating the median across all channels for each shank and subtracting this signal from each channel of the corresponding shank (Jun et al., 2017). Data were bandpass filtered with a fourth-order Butterworth filter $(0.3-6 \mathrm{kHz})$ for spiking activity. For further analysis, multiunit activity (MUA) was calculated by full-wave rectification, filtering with a sixth order low-pass Chebyshev-II filter (stopband attenuation of $50 \mathrm{~dB}$ ) and down sampling to $1 \mathrm{kHz}$.

\section{RF mapping and signal-to-noise ratio (SNR) analysis}

All data for the RF mapping experiments were recorded under head-fixation. Data were first cut into epochs of $280 \mathrm{~ms}$ (from $100 \mathrm{~ms}$ before to $180 \mathrm{~ms}$ after stimulus onset) based on the onset timing of stimulus presentation as determined from the photodiode signal. For incomplete trials (break fixation trials), a given epoch was included in the analysis as long as the eye position remained inside the fixation window throughout the epoch. To reject artifacts, we calculated the SD of MUA across time within each 280-ms epoch. Epochs in which the SD was 10-times larger than the median SD across all epochs were excluded from the analysis.

Recording sites were included in the analysis if the mean MUA from at least three different wedge stimuli and at least three different annulus stimuli evoked responses that were significantly larger than the MUA during baseline (paired t-test, alpha =0.01). The baseline was defined as the MUA 100-0 ms before stimulus onset.

For the calculation of RFs, MUA from 0 to $100 \mathrm{~ms}$ after stimulus onset from all artifact-free epochs was averaged, and this value was multiplied with the 2D matrix containing the intensity values from the images shown at the corresponding epoch, separately for annuli and wedge stimuli. All the resulting images were summed up and divided by a bias image to normalize for unequal repetitions of images. The bias image was computed by summing up all images that were presented in all artifact-free epochs, separately for annuli and wedge stimuli. The final RF map was calculated by pixel-wise multiplication of the two maps and then scaled to range between zero and one.

Estimates of RF size and position were obtained by fitting a Gaussian function to the annulus data and a von Mises function (the circular approximation of a Gaussian function) to the wedge data. Mean, and circular mean from the resulting model fits were used as peak eccentricity and polar angle, respectively. The RF size along the eccentricity axis $\left(\mathrm{RFS}_{\mathrm{e}}\right.$ ) was defined as the full width at half maximum (FWHM) of the Gaussian model fit. The RF size along the axis perpendicular to the eccentricity was calculated on the basis of the FWHM of the von Mises model fit: the resulting circular arc length was used to calculate the corresponding chord length $\left(\mathrm{RFS}_{\mathrm{C}}\right)$. The RF size was defined as the geometric mean of $\mathrm{RFS}_{\mathrm{e}}$ and $\mathrm{RFS}_{\mathrm{c}}$. 
For the analysis of SNR, RF maps were computed as mentioned above, but repeatedly from a subset of the data. For each subsampling run, we randomly picked a small number of stimulus presentations per condition from the complete dataset. This procedure was performed 100 times for each repetition size step from one to nine repetitions. Nine repetitions were chosen as the maximum for the analysis because it was the lowest number of available repetitions in the dataset. The analysis was performed by using the neuronal data after stimulus presentation (0-100 ms) and, as a baseline control, by using data before stimulus presentation $(-100-0 \mathrm{~ms})$. The SNR was calculated as the amplitude ratio of signal and noise in decibels $(\mathrm{dB})$. The signal was defined to be the mean value inside a region of interest $(\mathrm{ROI})$ on the RF map. The noise was defined as the mean value outside the same $\mathrm{ROI}$. The ROI was determined per recording site by thresholding the RF map that was calculated from all available data. Pixels with values larger than the half maximum of the RF map were defined as being within the ROI. For each recording site, the same ROI was used for all subsampling runs. Confidence intervals were calculated by bootstrapping $(10,000$ bootstrap replications) on the subsampled data. The mean SNR values and confidence intervals were averaged across animals and areas.

\section{Results}

\section{Eye tracking and behavioral setup}

We used a standard infrared-based eye-tracking system in combination with a custom calibration procedure to track the position of one eye of marmosets without head-fixation (Fig. 1A). Animals were trained to enter a tube-shaped chair and to position their head in front of a monitor without any immobilization of their body or head. The small size of the opening for the head allowed the animals to return to the same overall position and distance across behavioral sessions. Additionally, the reward-delivering lick spout was positioned centrally relative to the monitor, and animals quickly learned to keep their heads facing forward. This allowed successful tracking of pupil and corneal reflex as long as the animals were engaged in the task (Fig. 1B).

\section{Calibration}

In studies with human subjects and non-human primates, calibration of the eye signal is typically performed at the beginning of each experimental session. This is done by a short sequence of fixations at defined coordinates on the monitor. This approach consumes time within each session, and provides only one or few data points per target position. Therefore, we introduced a different approach that uses average data from several trials performed during an entire calibration session. The resulting calibration was then used in future sessions, without any further corrections or offset removal. Figure $2 A$ shows an example of such data after calibration. In a given calibration session, animals were required to briefly fixate a central fixation point and then saccade to a peripheral location indicated by a small stimulus (for details, see

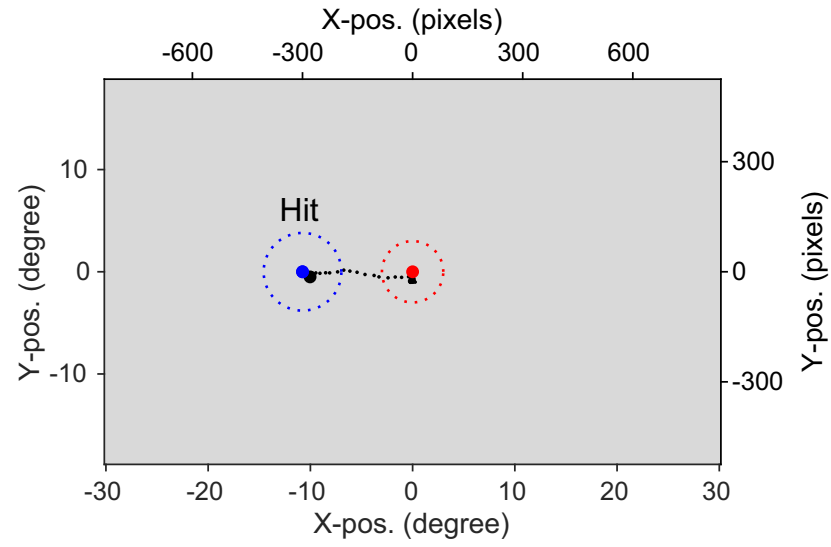

Movie 1. Head-free eye tracking during visual acuity measurements. Eye position and trial outcome from six example trials are shown in the movie (three hits and three miss trials). Red and blue dots indicate position of fixation point and target stimulus, respectively. Dashed circles are the corresponding eyetracking windows. Small black dots show the last $100 \mathrm{~ms}$ of eye position during the trials. Large black dot shows current eye position. [View online]

Materials and Methods). The recorded eye data were analyzed offline together with the known target and fixation locations to create a calibration template for future sessions. Target locations with the highest density were manually selected and used to fit a third-order 2D polynomial function. The resulting transformation function was applied to the horizontal and vertical component of the eye data. The corrected positional data can be seen in Figure $2 A, B$. Figure $2 C, D$ shows calibrated example saccades toward different target locations. The density of eye data was particularly high at the central location (Fig. 2B). This is because of the fact that animals were required to initiate trials by maintaining their gaze at the central fixation point. This provided a large amount of data from various trials.

The obtained calibration was used in subsequent sessions to assess visual acuity, and several example trials from one such session are shown in Movie 1.

\section{Accuracy and precision of central eye position}

Offline calibration might be prone to inaccuracies resulting from changes in the position of the animal within and between sessions. We quantified this by calculating offset and SD (referred to as 'sigma') for every session. We focused this analysis on the central 2.5 degrees and on the time from fixation onset until the animal made a correct saccade. This choice was motivated by the fact that the majority of studies use central fixation, and the presented procedure was primarily aimed to be used in such study designs. The density plots in Figures $3 A-C$ show example sessions from each of the four animals. The binned and averaged horizontal and vertical eye data (Fig. $3 A-C$, black dashed lines) were fitted with 1D Gaussians (Fig. $3 A-C$, red lines), and those were subsequently used to derive horizontal and vertical offset and sigma values (Guerrasio et al., 2010). 
A
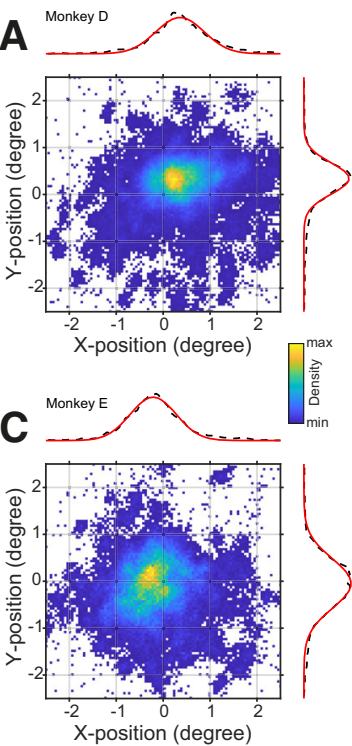

B
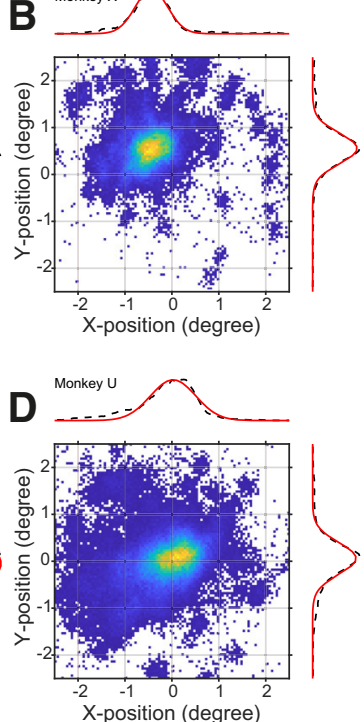

E
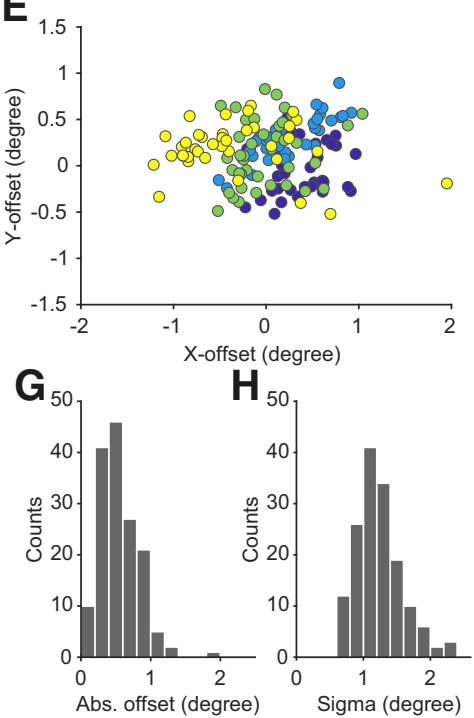

$\mathbf{H}_{50}$

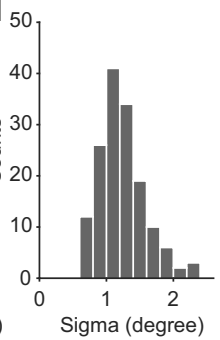

$\mathbf{F}$

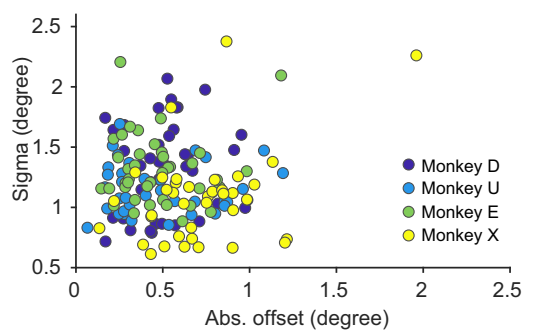

I

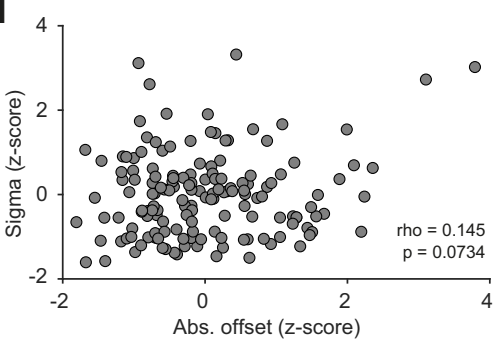

Figure 3. Quality of eye signals across sessions and animals. $\boldsymbol{A}-\boldsymbol{D}$, Example density plots of eye position during the visual acuity task for each monkey. Data are taken from time of fixation onset until correct response. Dashed lines show the average density for $X$ - and Y-positions. Red lines show Gaussian fits. $\boldsymbol{E}, \mathrm{X}$ - and Y-offset values for all sessions $(n=153$, color coded as indicated in panel $\boldsymbol{F}$ ). $\boldsymbol{F}$, Scatter plot of offset versus sigma values for all animals and sessions. $\boldsymbol{G}, \boldsymbol{H}$, Distributions of offset and sigma values for all sessions. I,Same as $\boldsymbol{F}$, but after z-transformation per animal. Eye signals from head-fixed animals are presented in Extended Data Figure 3-1.

Figure $3 E, F$ shows the resulting offset and sigma values, respectively, for all 153 analyzed sessions. Overall, offset positions appear to spread around the fixation point. However, individual animals seem to form clusters (for example Monkey $\mathrm{X}$ in the top left direction), indicating small systematic biases at the level of individual animals (see Discussion). Most sessions showed an absolute offset that was below 1 degree (median $=0.503 \pm 0.29$ degree) and a sigma of $<2$ degrees (median $=1.17 \pm 0.34$ degree). As expected, these values were larger than measurements obtained during head-fixation (Extended Data Fig. 3-1A-D; median of absolute offset $=0.177 \pm 0.068$ degree; median of absolute sigma $=0.430 \pm 0.035$ degree; Monkey $\mathrm{D}: n=9$ sessions; Monkey A: $n=8$ sessions).

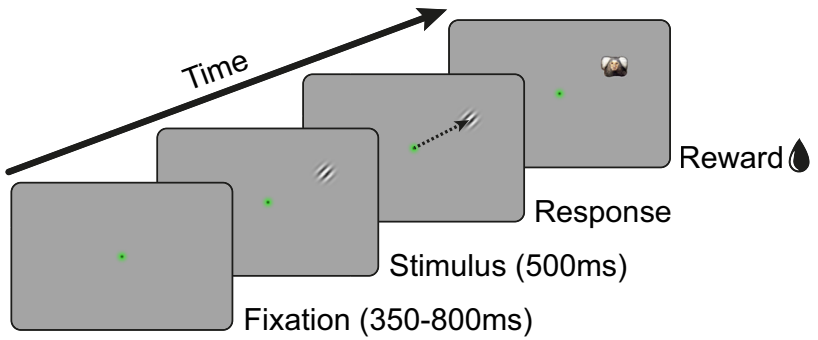

Figure 4. Visual acuity task design. Animals were required to fixate a central fixation point for $350-800$ ms. A small Gabor stimulus ( 0.3 degree, $50 \%$ contrast) with variable spatial frequency was presented for $500 \mathrm{~ms}$ randomly at one of eight possible locations (10.77 degrees eccentricity). After a correct saccade to the target, a picture of a marmoset face was displayed, and the animal was rewarded.
It is possible that the sessions with the largest offset would have the largest SD. This might be expected in sessions in which the position of the animal is not optimal. To quantify this, we calculated the correlation between absolute offset and sigma values. To avoid any spurious effects of potential across-subject correlations, the offset and sigma values were first $z$-transformed per animal, and then combined before the correlation analysis (Fig. 3). There was no significant correlation (Pearson's rho $=0.145, p=0.0734^{\mathrm{a}}$, see Table 1 for details), indicating that sessions with a large offset could still result in a reliable but shifted estimate of the eye position around the fixation point.

\section{Visual acuity in head-free marmosets}

In order to test whether the head-free eye-tracking quality is sufficient for visual neuroscience applications, we trained animals to perform a visual acuity task (Fig. 4). Animals could initiate trials by moving their gaze to a central fixation point. After $350-800 \mathrm{~ms}$ of fixation, a small Gabor stimulus (50\% contrast) with variable spatial frequency appeared randomly at one of eight equi-eccentric locations. Trials were categorized as hits if the animal made a saccade to the target stimulus within $500 \mathrm{~ms}$.

At the time of visual acuity measurements, all animals were familiar with simple face-detection tasks (Fig. 1A) and calibration tasks (Fig. 2) and had no problem switching to the visual acuity task. Animals performed on average $\approx 100$ hits per session. We fitted RTs and hit rates, as functions of the spatial frequency, with a four-parameter logistic function, separately per animal (Fig. 5A). Figure 5B shows the underlying $R T$ distributions from hit trials across all conditions and sessions. 
A
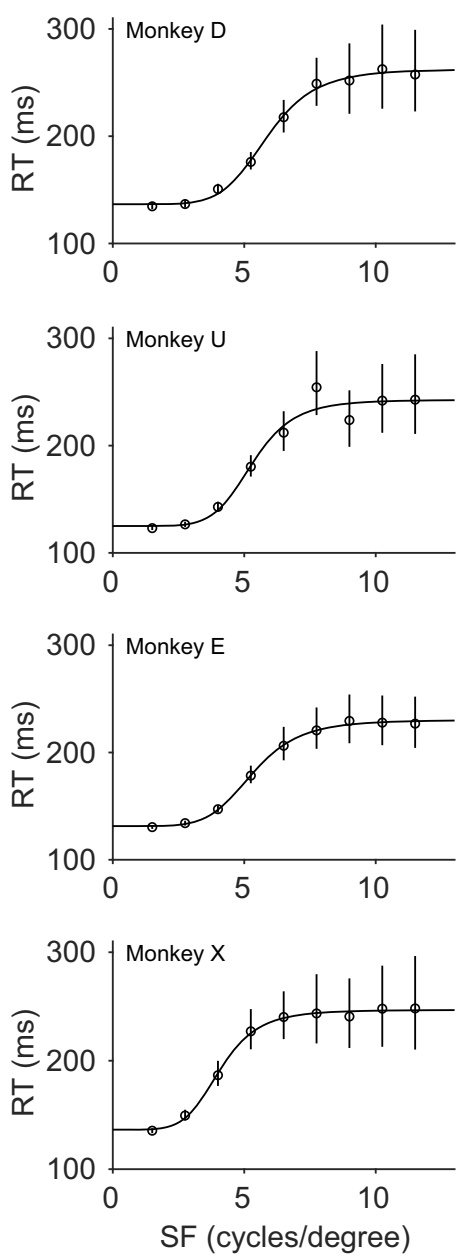
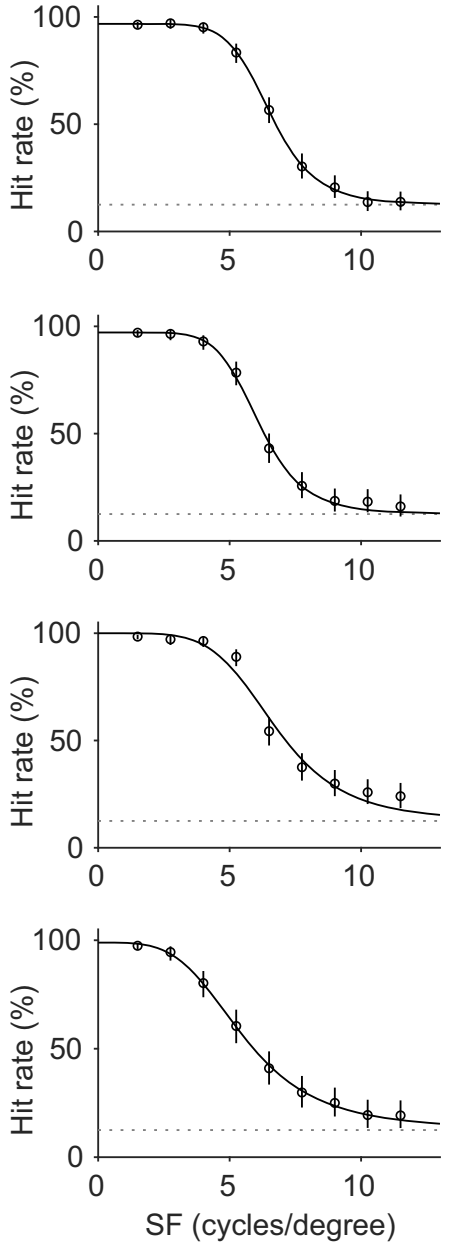

B
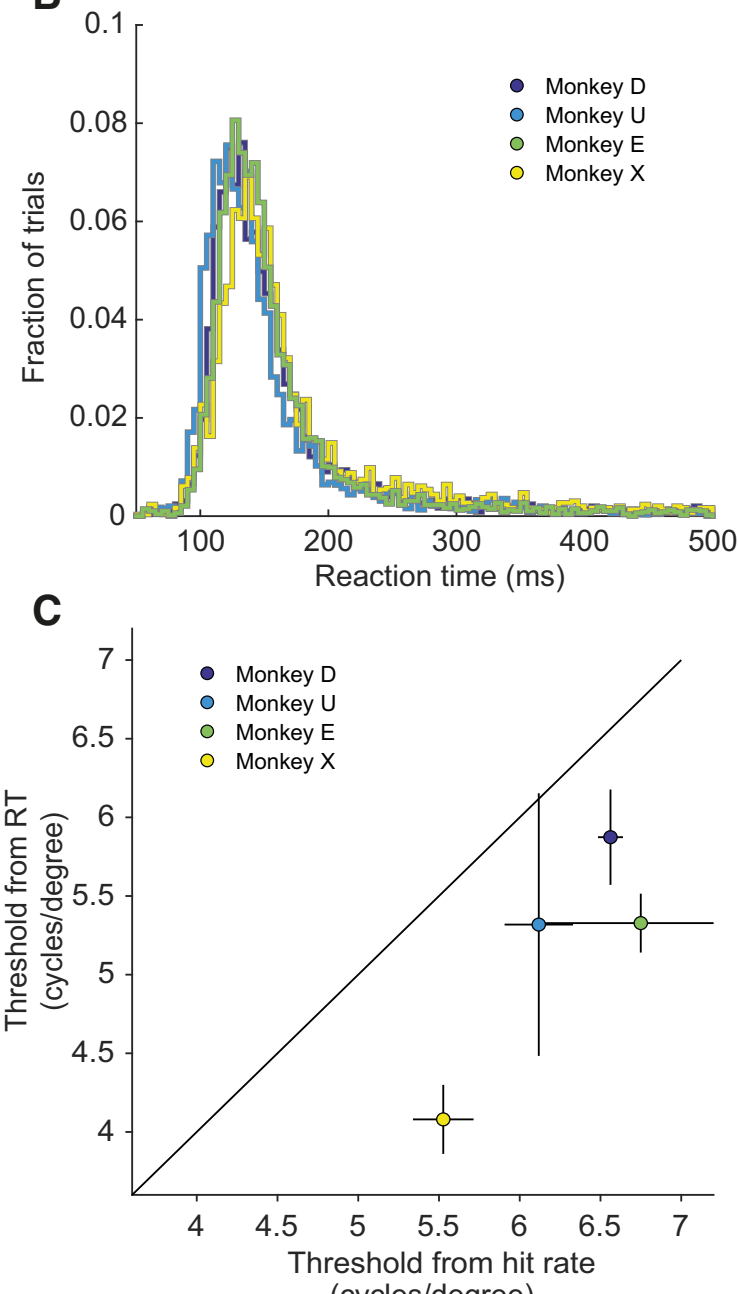

Figure 5. Visual acuity measurements in head-free marmosets. $\boldsymbol{A}, \mathrm{RT}$ and hit rate as a function of stimulus spatial frequency (SF) for each of the four animals. Mean RTs are shown on the left panel, and hit rates are shown on the right panel. Error bars indicate $99.9 \%$ confidence intervals. In the hit rate plots, dotted lines show expected chance level performance of $12.5 \%$. B, RT distributions, per animal, pooled over all conditions and sessions. C, Comparison of acuity thresholds calculated from RTs and hit rates. Error bars indicate $95 \%$ confidence intervals.

RT values were longer for higher spatial frequencies: the average upper asymptote $(245.5 \pm 6.6 \mathrm{~ms}$, mean \pm SEM) was significantly above the lower asymptote $(132.4 \pm 2.7 \mathrm{~ms} ; p=$ $0.00028^{\mathrm{b}}$, paired t-test across the four animals, see Table 1 for details). For the fits of the hit rates, the asymptotic value for high spatial frequencies was fixed to the chance level of $12.5 \%$. The parameter that determines the inflection point of the psychometric function corresponds to the perceptual threshold. We calculated thresholds from hit rates and from RTs for each animal. As expected, thresholds were higher, corresponding to a higher visual acuity, when calculated from hit rates $(6.4 \pm 0.3$ cycles/degree, mean \pm SEM) as compared with RTs (5.2 \pm 0.4 cycles/degree, mean \pm SEM), indicating a more accurate estimation (Fig. 5C; $p=0.012^{C}$, paired t-test across the four animals, see Table 1 for details). The thresholds obtained from hit rates are in line with previously reported values from Nummela et al.
(2017) under head fixation. This confirms that it is possible to measure visual acuity in marmosets without head-fixation.

\section{Efficient RF mapping}

Having established that marmosets can be behaviorally assessed without head fixation, we turned our interest to another important aspect of visual neuroscience, namely the mapping of visual RFs. As was the case for our headfree psychophysics experiments, the goal was to adapt a technique for a fundamental aspect of visual neuroscience to make it more suitable for the marmoset. Because of the small size of RFs in early visual areas, animals were head-fixed for the recordings (however, see Discussion).

Commonly used stimulus types for RF mapping are: spatial noise (Citron and Emerson, 1983; Niell and 


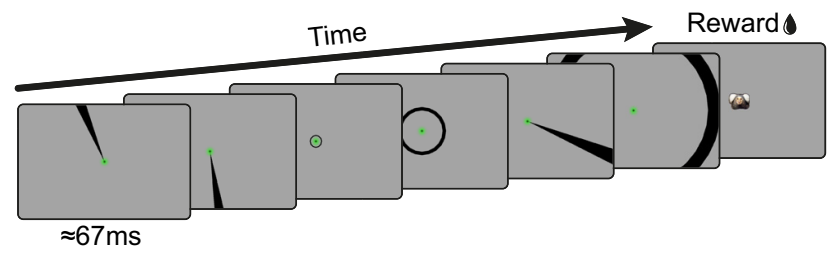

Figure 6. RF mapping task. Annulus and wedge stimuli with sizes and orientations that covered the whole monitor were flashed for eight frames $(\approx 67 \mathrm{~ms})$ each. The animal was required to maintain fixation throughout the trial and was rewarded at the end. Correct trials were signaled by the appearance of a marmoset face at the center. Typically, nine to ten stimuli were flashed per trial, but only six are shown here for clarity.

Stryker, 2008), flashing dots or squares (Jones et al., 1987; Tolias et al., 2001; Martinez et al., 2005), and moving bars (Hubel and Wiesel, 1962; Fiorani et al., 2014). Another possibility is the use of rotating wedges in combination with expanding and contracting annuli. The latter is often used in fMRI studies (Sereno et al., 1995; Benson et al., 2018). Moving bars and rotating wedges are efficient stimuli because of their spatial correlation. However, because they are very salient, it can be challenging for animals to maintain their gaze at the fixation point. We developed a novel approach that uses fast flashing annuli and wedges to cover a large part of the visual field, thereby being very efficient. Importantly, the presented stimuli are flashed for a short duration at a random location and are either symmetric to or connected to the fixation point. We reasoned that this design might make it easier for the marmosets to maintain fixation.

\section{Annulus-and-wedge RF mapping task}

Stimuli were black wedges and annuli, presented on a gray background (Fig. 6). Wedges subtended 9 degrees of polar angle and were presented in steps of 4.5 degrees of polar angle. Annuli had a width that corresponded to the width of the wedge at the eccentricity of the midpoint of the respective annulus. As soon as the animal moved its gaze to the central fixation point, a sequence of nine to ten stimuli was flashed within a typical trial. Each stimulus presentation lasted for eight frames at a monitor refresh rate of $120 \mathrm{~Hz}$, resulting in a presentation time of $\approx 67 \mathrm{~ms}$. At the end of a correct trial, animals were rewarded, and a marmoset face indicated the end of the trial. In case the animal broke fixation before the full stimulus sequence was over, we continued presenting the stimulus sequence and no reward was given. This was done to let the animal freely explore the stimuli to learn that there was no reward associated with looking at them.

In order to test whether the RF mapping stimuli could be used to efficiently locate the positions at which neuronal activity was highest (i.e., location of the classic RF center), we recorded spiking activity from areas $\mathrm{V} 1$ and V6 (Fig. 7A). Animals had no problem maintaining fixation throughout many trials, and successfully completed several hundred stimulus presentations in short recording sessions (data from Fig. $7 ; n=1654$ in $\approx 11 \mathrm{~min}$ for
Monkey $\mathrm{D}$ and $n=2529$ in $\approx 12 \mathrm{~min}$ for Monkey A; corresponding to 9-17 and 14-24 repetitions per stimulus condition, respectively, after artifact rejection). We observed spontaneous neuronal activity on several recording sites as well as clear stimulus-evoked activity (V1 example trace in Fig. $7 A)$. In total, 188 out of $384(\approx 49 \%)$ sites were significantly modulated from baseline and selected for further analysis (Monkey A: 63 of 64 sites in V1 and 60 of 128 sites in V6; Monkey D: 58 of 64 sites in V1 and 7 of 128 sites in V6).

We calculated the mean MUA for all presented annuli and wedges separately, which resulted in activity profiles across all tested eccentricities and polar angles (Fig. 7C,F, black lines). To estimate RF size and position, we fitted a Gaussian function or its circular approximation (von Mises function) to the annulus and wedge data, respectively (Fig. 7C,F, red lines). The extracted RF sizes in V1 and V6 were largely consistent with previously published data from anesthetized animals (Rosa and Schmid, 1995; Rosa et al., 1997; Chaplin et al., 2013; Yu et al., 2020), although V1 RFs appeared slightly larger (Extended Data Fig. 7-1A; see also Discussion). Based on the electrode target coordinates, the RF locations from V1 were expected to be in the lower visual field, relatively close to the vertical meridian (Chaplin et al., 2013). V1 RFs from both monkeys were consistent with this (Extended Data Fig. 7-1B,D), suggesting placement in area V1. In V6, RFs spread across the upper and lower visual field, consistent with the compressed retinotopic representation of this area (Yu et al., 2020). Furthermore, as expected from the retinotopic organization of both areas, RFs from nearby electrode sites showed similar RF locations (Extended Data Fig. 7-1B-E).

In order to relate spiking activity to the visual space across the monitor, we applied a reverse correlation technique (Jones et al., 1987; Ringach, 2004), that resulted in two activity maps (for details, see Materials and Methods). Each map shows the location of those annuli or wedges, respectively, that evoked the highest spiking activity in the respective recording site (Fig. $7 D, G)$. The two maps were then combined by multiplication to reveal the peak RF location of the recorded neurons. Maps from the model fits were obtained by transforming the model data from polar coordinates to Cartesian coordinates for each pixel of the monitor (Fig. 7E,F).

To quantify the efficiency of our RF mapping technique, we calculated the SNR for RF maps from a subset of the data. Subsampling was performed by randomly picking a small number of stimulus presentations per condition from the full dataset. This procedure was performed 100 times for each step, from one to nine repetitions. SNR was calculated as the amplitude ratio in decibels $(\mathrm{dB})$ of the mean values inside and outside a ROI on the RF map. The ROI was determined by thresholding the RF maps that included all available data at the half maximum (for details, see Materials and Methods). Figure $8 A$ shows example RF maps calculated from a random small subset of stimulus presentations. The RF center position is already visible with one or two repetitions per condition and becomes clearer with more repetitions. As a baseline control condition, we 


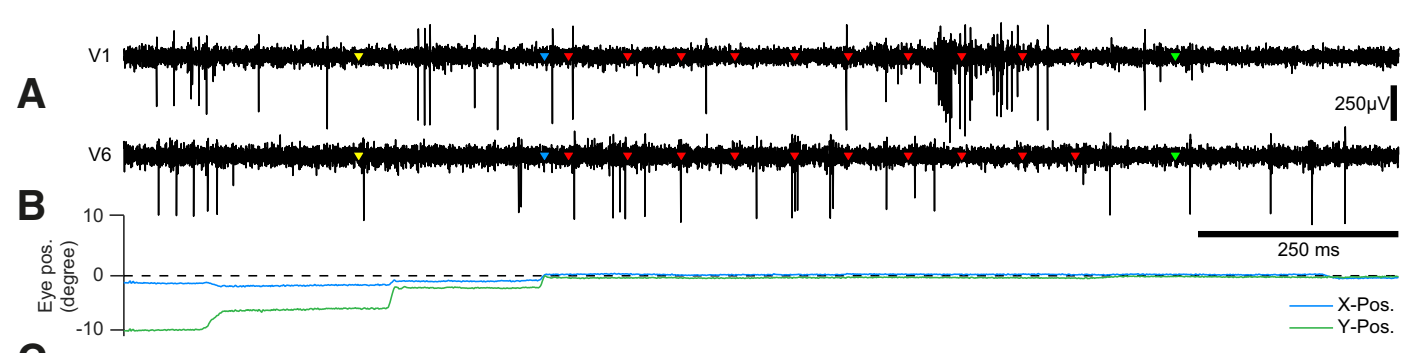

C
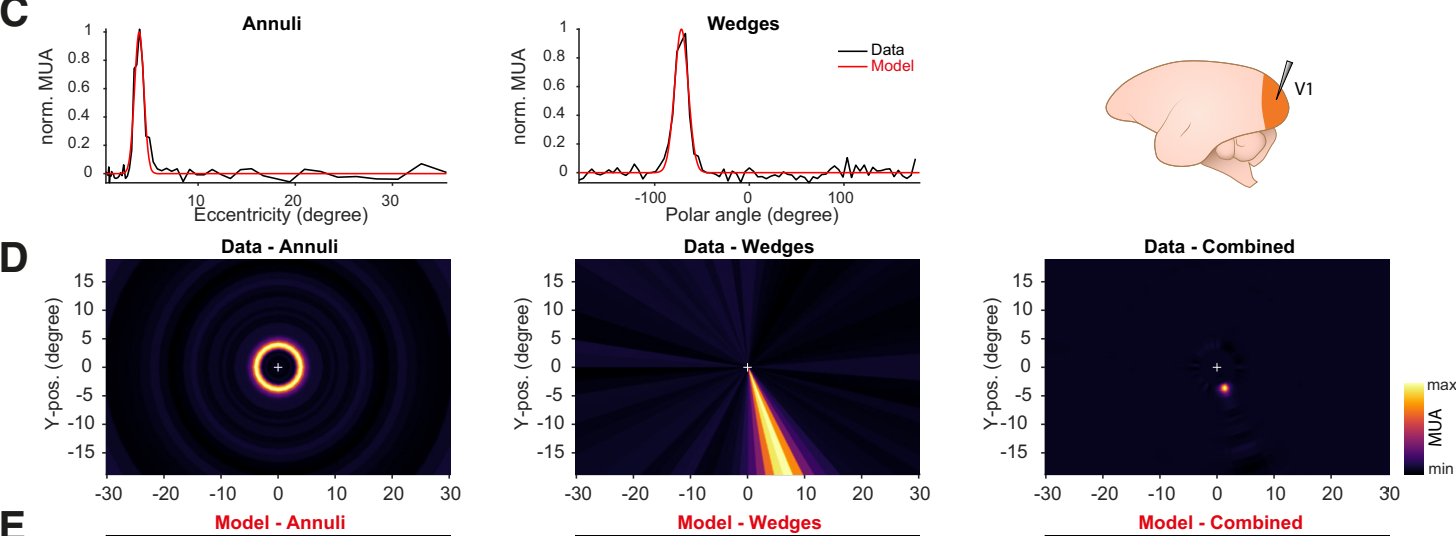

E
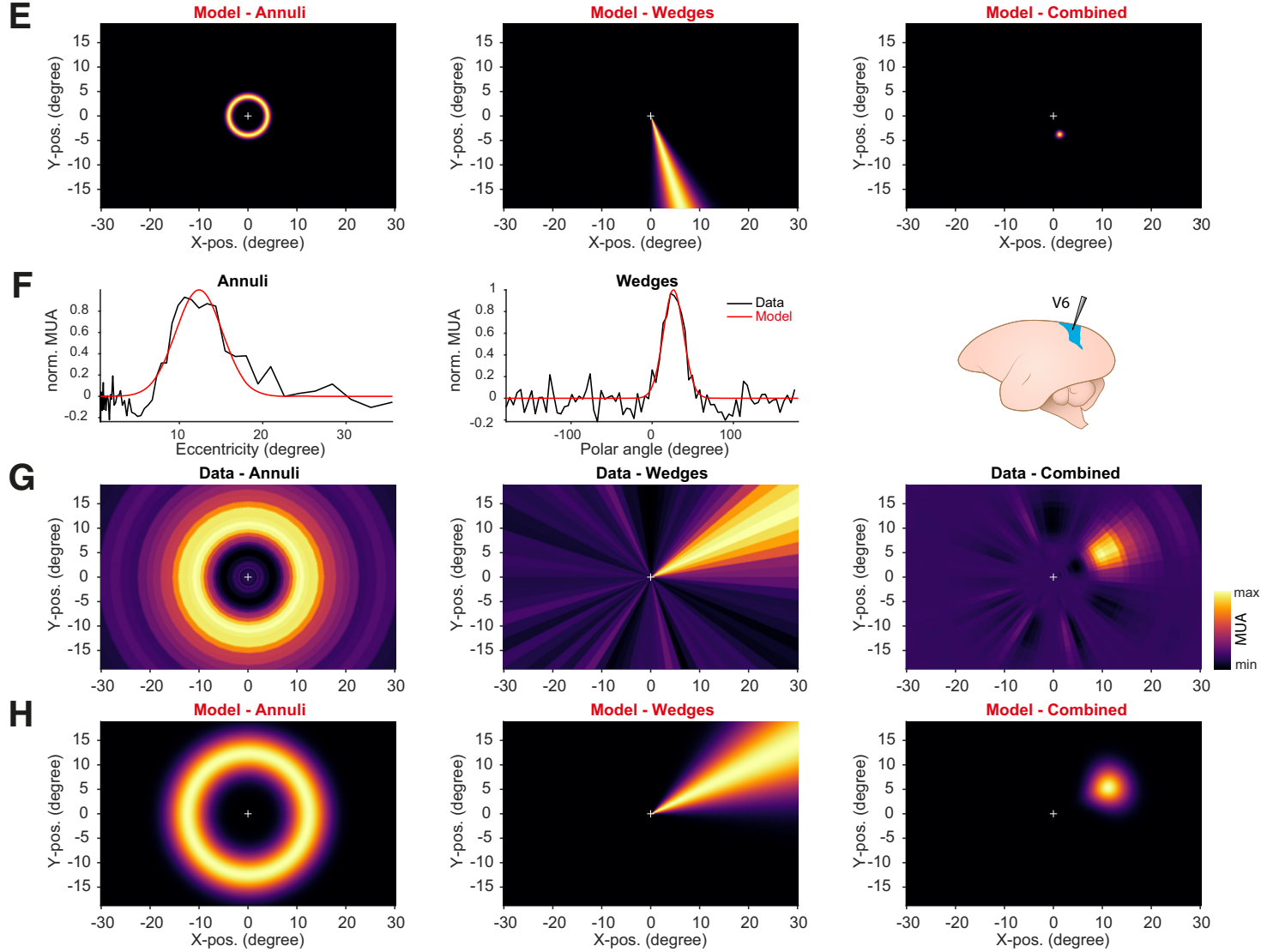

Figure 7. RF mapping results. $\boldsymbol{A}$, Spiking activity from two example channels simultaneously recorded from cortical areas $\mathrm{V} 1$ and V6 in Monkey A. Yellow markers indicate start of the trial. Blue markers indicate fixation onset. Red markers indicate onsets of individual stimuli. Green markers indicate time of reward. B, Corresponding eye trace for example trial. Note fixation onset as indicated by blue makers. C, MUA profiles and model fits for all presented annuli and wedges from an example recording site in area V1. $\boldsymbol{D}$, Reverse correlation RF maps across the monitor, from annulus, wedge, and combined MUA data. Color indicates normalized multiunit response. White cross indicates position of fixation point at the center of the monitor. $E$, RF maps across the monitor, from annulus, wedge, and combined model data. $\boldsymbol{F}-\boldsymbol{H}$, Same as $\boldsymbol{C}-\boldsymbol{E}$ but from recording site in area V6 in Monkey D. RF sizes and positions of all stimulus-driven recording sites are presented in Extended Data Figure 7-1. 
A

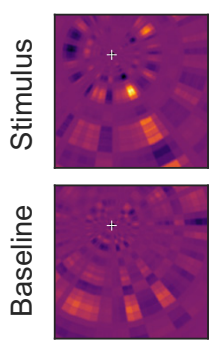

$\mathrm{n}=1$
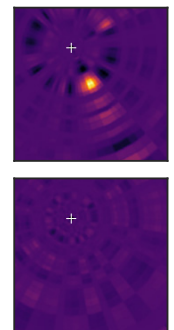

$n=2$

B

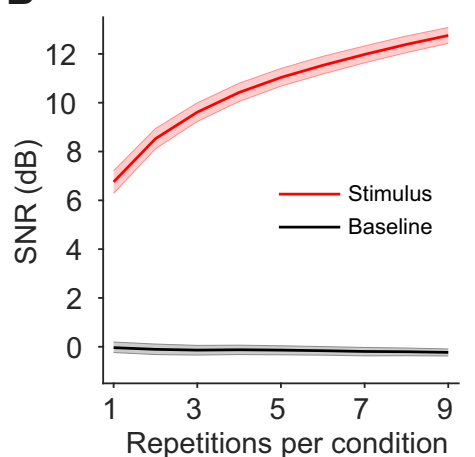

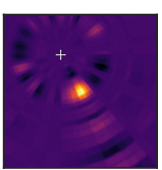

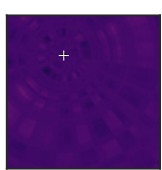

$n=3$
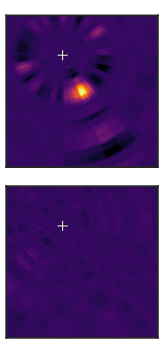

$n=4$
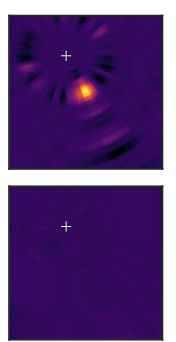

$\mathrm{n}=5$
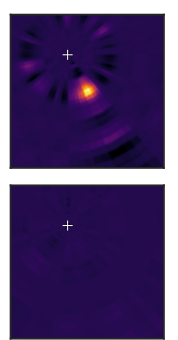

$n=6$
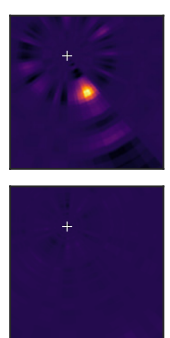

$\mathrm{n}=7$
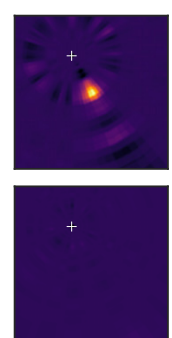

$\mathrm{n}=8$

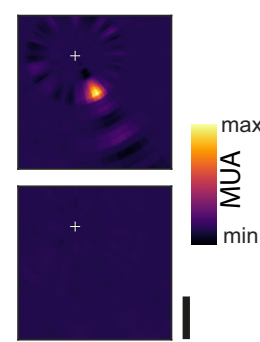

$\mathrm{n}=9$

\section{C}

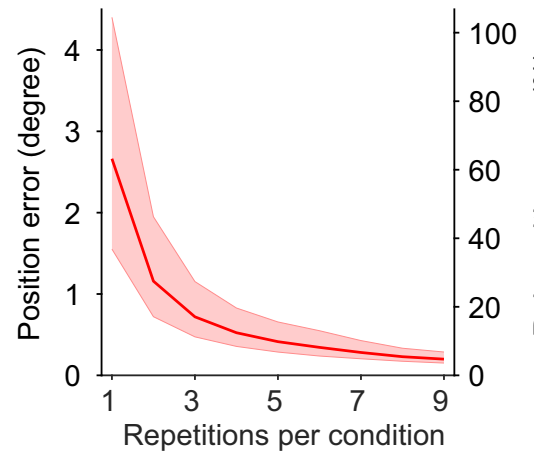

D

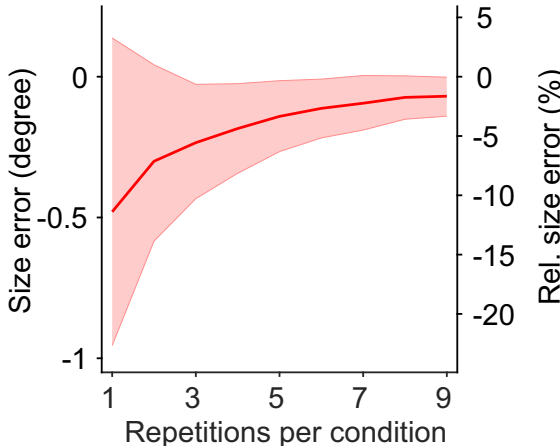

Figure 8. Efficiency of RF mapping. $\boldsymbol{A}$, Example RF mapping results from subsampling procedure for up to nine repetitions per stimulus condition. Data from example recording site in Monkey D. RF location is already visible with a very low number of repetitions. White cross indicates position of fixation point at the center of the monitor. Color indicates normalized multiunit response. Scale bar: 5 degree. B, SNR from subsampling procedure of RF mapping data, averaged across recordings sites from both monkeys $(n=188)$. Reverse correlation analysis was performed from neuronal data following the stimulus presentation (red line) and, as control, preceding the stimulus presentation (black line). Position error $(\boldsymbol{C})$ and size error $(\boldsymbol{D})$ from subsampling procedure of RF mapping data, averaged across all recording sites from both monkeys. Shaded regions indicate $99.9 \%$ bootstrap confidence intervals.

also calculated RF maps from the stimulus-unrelated activity preceding the stimulus. The resulting RF maps show no spatially specific activation, because of the MUA being uncorrelated with the future stimulus. Accordingly, the SNR for the baseline condition remains flat, even when the number of repetitions increases (Fig. 8B). In contrast, the SNR calculated from stimulus-evoked MUA increases with the number of repetitions. Crucially, SNR values were already high when a small number of repetitions was used.

Next, we quantified the absolute and relative errors in the estimation of RF position and size. We used the same subsampling procedure as in the SNR analysis and repeatedly calculated position and size of the resulting RFs with varying amounts of data. We then compared the subsampled results to the values obtained by using all available data. Relative errors were calculated by normalizing the absolute error to the size of the corresponding RF obtained from all available data. The errors for position and size converged quickly toward zero with relatively few repetitions per condition (Fig. $8 C, D$ ). The negative values for size errors reflect an initial underestimation of the size for small repetition numbers.

These results confirm that our annulus-and-wedge RF mapping technique is a very efficient way of mapping visual RFs. It yields reliable results with fewer than 10 repetitions per stimulus condition, which can be obtained in 5-10 min of recording time.

\section{Discussion}

The goal of this work was to develop two key methodological advancements for visual neuroscience that are designed to be more suitable for the marmoset monkey. First, we showed that head-free eye tracking in marmosets can be achieved with existing hardware. The analysis of accuracy and precision revealed that head-free eye tracking is potentially suitable for a variety of visual neuroscience applications. We tested the applicability by measuring visual acuity in four head-free marmosets and demonstrate that the obtained acuity thresholds are consistent with previously published data obtained under head fixation (Nummela et al., 2017). Second, we introduced a novel method for efficient RF mapping that does not rely on moving stimuli, but uses a sequence of rapidly flashing annuli and wedges. To validate the novel RF mapping technique, we recorded data in areas V1 and V6 and show that RF locations are readily obtained in a short time and with very few stimulus repetitions.

\section{Limitations and advantages of head-free eye tracking and offline calibration}

As expected, the accuracy and precision obtained from head-free animals is lower than what can be achieved under head fixation (Extended Data Fig. 3-1A-D). Some of the imprecision can be explained by our choice of lens that was not optimized for maximum magnification of the 
pupil into the field of view (Fig. 1B). Instead, the lens was chosen to have a large field of view (i.e., a large "head box") that allowed capturing pupil and corneal reflex signals despite head displacement. A large part of the inaccuracy arises most likely from movement of the animals within a session and across sessions. In principle, these offsets and errors from the resulting head-rotation could be partly compensated by tracking the animals' head position in real time (SR Research, 2009; Niehorster et al., 2018). However, this can require the placement of a physical tracking target on the subject (Wang et al., 2019), which might be difficult to apply when working with small animals. More complex, model-based algorithms for head tracking (Yang and Zhang, 2002) might resolve this, and commercial systems (albeit optimized for humans) do already exist (Niehorster et al., 2018). The approach presented here focused on the implementation of a procedure with minimal hardware and software changes. It can therefore be used within the configuration range of existing commercial eye trackers in many laboratories.

Inaccuracies in eye position measurements might influence the outcome of a study (Holmqvist et al., 2012; Hessels et al., 2016). It was therefore crucial to validate our approach against previously reported data. The task described here was used because it had been successfully deployed before in head-fixed marmosets to assess visual acuity (Nummela et al., 2017). This allows for a close comparison of results and confirms that it was possible to measure visual acuity thresholds without the need for head fixation. It should be noted however that small differences in the experimental setup (i.e., monitor specifications, luminance, and distance to the screen) could still result in different acuity thresholds. We also found that acuity thresholds were higher, corresponding to a higher visual acuity, when calculated from hit rates (Fig. 5C). This confirms that hit rates are the preferred measurement for the estimation of perceptual thresholds (Palmer et al., 2005).

As mentioned above, offset values from individual animals appeared to cluster around a non-zero value across sessions (Fig. 3E), e.g., sessions from Monkey X seemed to cluster in the top left direction. Such clustering potentially indicates small biases during the calibration session. If so, offsets might be further reduced by subtracting the average offset across all recorded sessions, and thereby post hoc recalibrating the data. This could also be used to implement an iterative procedure that uses every recorded session to optimally compensate for offsets.

There are however limitations to the precision that can be achieved with the head-free eye-tracking approach presented here. The median absolute offset across sessions (analyzed within the central 2.5 degrees) was $0.503 \pm 0.29$ degree, the median SD (sigma) was $1.17 \pm 0.34$ degree. These values indicate that it would not be feasible to perform experiments requiring continuous high-precision eye-position control. Nevertheless, the data quality is sufficient for many psychophysics applications (see below) and might even be used in experiments in which neuronal recordings are made from brain areas that have large RFs and exhibit translation invariance, such as the inferior temporal cortex (Tovee et al., 1994; Rolls et al., 2003).

We used nine calibration points and a polynomial function to calibrate eye data. The calibration function relies on interpolation between those points and extrapolation beyond the points. Therefore, care should be taken about the choice of the calibration points and the interpretation of eye data beyond the calibration points. Our procedure made use of the relatively long fixation times at the central fixation (Fig. 2B). This sampled eye data across varying head positions, and thereby resulted in a robust estimate for the calibration around the central location. In the same way, one could optimize the calibration positions to match the future positions for any task, thereby minimizing the effects of interpolation errors. Practically, this would entail that the very stimulus positions used in the actual task are also used for calibration. Furthermore, increasing the number of calibration targets or using smooth pursuit eye data can improve accuracy (Pfeuffer et al., 2013; Kasprowski et al., 2014; Hassoumi et al., 2019).

Head movements during the task will influence eyetracking quality and can result in loss of signal (Niehorster et al., 2018). The eye tracker used in this study allows for a maximum of $\pm 25 \mathrm{~mm}$ horizontal or vertical head movement without accuracy reduction (SR Research, 2009). The horizontal limits and the upper vertical limit are most likely not reached because the small opening for the head does not allow such large movements. From our observations, we estimate the actual possible head movement to be approximately $\pm 15 \mathrm{~mm}$. The lower vertical limit might be reached when the animal is retracting its head partly into the chair. Other movement types (i.e., yaw, roll and pitch) will also contribute to the reduction of signal quality and have been described in detail for human subjects (Ehinger et al., 2019). Furthermore, head-movement related changes in the distance between the eye and the camera can result in erroneous changes in calibration gain. An increased gain will make it more difficult for the animal to maintain fixation. It might also result in "overshooting" when executing a saccade to the target position. A gain-increase can therefore result in more aborted trials ("break fixation" trials) and in fewer correct trials because of overshooting. In the case of a gain decrease, maintaining fixation might be easier for the animal, because of the apparently lower amplitude of eye movements. However, correct execution of a saccade to the target position might be impaired because of "undershooting" arising from the lower gain. In general, undershooting or overshooting are not likely to cause large changes in trial outcome because the target tracking windows used in this study are relatively large (3-4 degrees radius). Importantly, such changes would also affect the easiest conditions, thereby decreasing the upper asymptote of the psychometric curves. However, all animals in this study have a hit rate close to $100 \%$ for the easiest stimuli (Fig. 5A). This is an indication that there was no strong influence resulting from changes in gain that could arise from the positional changes of the animals. 
Table 1: Statistical tests reported in Results, labeled in the text by the letters in the left-hand column

\begin{tabular}{llllllll}
\hline & Distribution & Type of test & Statistic & $p$ value & Power & Sample size & Animals \\
\hline a & Non-normal & Pearson correlation & rho $=0.145$ & $p=0.0734$ & 0.435 & $n=153$ & $n=4$ \\
b & Normal & t-test (paired, two-sided) & T $=-19.803$ & $p=0.00028$ & $\approx 1$ & $n=4$ & $n=4$ \\
c & Normal & t-test (paired, two-sided) & T $=5.427$ & $p=0.012$ & 0.935 & $n=4$ & $n=4$ \\
\hline
\end{tabular}

Changes in the distance between the eyes and the monitor, i.e., depth movement, would also lead to a change in stimulus position and size on the retina of the animal. An increased distance would result in lower eccentricity and could thereby increase the animals' detection performance. At the same time, it would result in decreased angular stimulus size, thereby counteracting the performance increase to some extent. Since we did not measure head-position data, we cannot quantify how this might have influenced our results. However, we can provide an estimate on the expected maximum change in stimulus eccentricity and size, based on the geometry of our setup. Because of the small opening for the head and the position of the lick-spout in front of the animal, we estimate the actual depth movement to be smaller than $\pm 5 \mathrm{~mm}$. Under the very conservative assumption of $\pm 10 \mathrm{~mm}$ depth movement, the calculated difference in stimulus eccentricity is \pm 0.23 degrees. The resulting change in angular stimulus size is \pm 0.024 degrees, in the opposite direction. Considering that stimuli are presented in the visual periphery (at 10.77 degrees), where eccentricity-dependent changes in visual acuity are not as steep as in the fovea, the influence of depth movements on our results are expected to be very small.

We focused our analysis mainly on the eye data around the central fixation location. This was primarily done to reduce the influence of variance arising from the behavior of the animals. Although we did not measure this explicitly, it can be expected that head movements are being executed when the animals need to position their gaze on a peripheral target (Pandey et al., 2020). Without head movement, marmoset eye position is most of the time within the central 5-10 degrees (Mitchell et al., 2014). Therefore, peripheral eye data, which is not precisely corrected for head position, should be interpreted with care. Yet, a large proportion of visual psychophysics studies do not require high accuracy eye-tracking in the visual periphery, but only in the center (Carrasco, 2011; AntonErxleben and Carrasco, 2013). The visual acuity task presented here is an example of such a task design. Target locations are far enough apart and their respective target windows can be relatively large, thereby allowing the animal to perform the task even under noisy conditions (see also Movie 1). However, it is still important to track eye position reliably during the fixation period to prevent the animal from moving its gaze closer to the target. Such a change in eye position would bring the stimulus closer to the fovea, which in turn would result in a higher visual acuity (Chaplin et al., 2013; Nummela et al., 2017). This potential confound is additionally controlled for by using a randomized and symmetrical stimulus arrangement (Fig. 4).

The use of head-free eye tracking can provide several advantages. It enables the investigation of behaviors that are difficult or impossible to be studied under head fixation. Sound localization for example is strongly impaired during head-fixation (Populin, 2006) and the natural pattern of eye movements can be disrupted in some animals (Wallace et al., 2013; Meyer et al., 2020). Other behavioral tasks per definition require an unconstrained animal and can at best be approximated in virtual environments, i.e., tasks related to navigation, complex movement, social interactions and foraging. A further advantage of our approach is that it can be used in completely naive animals, without the need for implantation of a head-post. This could be used for screening animals before implantation to select individuals with normal acuity (Graham and Judge, 1999) and overall good behavioral performance. Additionally, naive animals can be pretrained for complex tasks with an automatic home-cage training setup (Calapai et al., 2017; Berger et al., 2018), thus potentially increasing the number of animals in a study.

Under voluntary semi-automatic conditions, marmosets work reliably but for relatively short amounts of time (Walker et al., 2020), thus making every minute of recording time very valuable. We show that it is possible to perform psychophysical measurements without spending time on daily re-calibration. This reduces the stress for both the animal and the experimenter and increases data collection time. Future work might make use of fully automated experimental setups (Poddar et al., 2013), with multicamera tracking (Young et al., 2016) and advanced 3D pose estimation (Mathis et al., 2018; Nath et al., 2019), potentially in combination with wireless neuronal recordings (Eliades and Wang, 2008; Roy and Wang, 2012; Courellis et al., 2019).

\section{Limitations and advantages of annulus-and-wedge RF mapping}

RF mapping techniques are often optimized for specific requirements and scientific questions. Stimuli such as spatial noise patterns (Citron and Emerson, 1983; Niell and Stryker, 2008) and to some extent flashing dots or squares (Jones et al., 1987; Tolias et al., 2001; Martinez et al., 2005) can be used to infer detailed spatiotemporal RF characteristics (Ringach, 2004). However, because of the large number of possible stimulus configurations, such approaches can be time-consuming when RF centers need to be localized across large parts of the visual field. Moving bars (Hubel and Wiesel, 1962; Fiorani et al., 2014) and moving annulus-and-wedge stimuli (Sereno et al., 1995; Benson et al., 2018) are more suitable for that purpose because of their spatial structure. The stimulus design presented here was motivated by two factors: the behavioral requirements of the marmoset and the experimental requirement to locate RF centers with unknown positions and sizes across two visual areas. We initially 
presented some animals with moving bars and observed that they would break fixation and follow the bar movement on almost every trial, thereby making data collection nearly impossible. This comes as no surprise given that marmosets are prey animals and that it is essential for their survival in the wild to detect potential predators (Ferrari, 2008).

The flashing annulus-and-wedge RF-mapping approach combines several properties that are advantageous for the localization of RF centers with little data. The time for a single stimulus presentation is short ( $\approx 67 \mathrm{~ms})$, and the set of stimuli covers the entire monitor. This enables mapping of a relatively large part of the visual field within a short duration. The design of annuli and wedges corresponds to a polar coordinate system. Every position on the monitor will at some point display a wedge with a specific polar angle and an annulus with a specific eccentricity. In this way, for each neuron, or MUA, the polar angle and eccentricity for which it shows the maximum response can be determined (Fig. $7 \mathrm{C}-H$ ). Yet, the stimulus set does not cover all orientations equally at every position. This might lead to a reduced response in case neurons are not optimally tuned to the annuli and wedges shown in their respective RFs. This limitation could be addressed by filling the stimuli with textures of randomized orientations.

One additional feature of the design is the stimulus scaling with eccentricity. The increasing RF size with eccentricity because of cortical magnification is a wellestablished phenomenon (Harvey and Dumoulin, 2011). This is one of the reasons why rotating wedges and contracting/expanding annuli are typically used in fMRI experiments where it is necessary to stimulate large visual cortical regions across eccentricities (Sereno et al., 1995; Benson et al., 2018). A related approach has been used by Hung et al. (2015) to map the foveal bias of visual areas with fMRI in the marmoset, albeit without strict eye fixation and the lack of appropriate stimulus resolution. In contrast, bar stimuli with a fixed width will be suboptimal both in driving neurons that have small parafoveal RFs and neurons that have large peripheral RFs. An RF-mapping stimulus that takes eccentricity into account will elicit stronger and more evenly distributed neuronal responses across the cortex.

The reported errors for RF position and size estimates were found to be small even after few repetitions per stimulus condition (Fig. 8C,D). RF positions and sizes from both areas were largely consistent with previously published data (Extended Data Fig. 7-1A-E). Notably, the RF size estimates from area $\mathrm{V} 1$ appear to be $\approx 0.5-1$ degree larger than previously reported in anesthetized marmosets (Rosa et al., 1997). This discrepancy is most likely because of the fact that our results are obtained from awake animals that execute small eye movements (micro-saccades) during the fixation period. Additionally, our results are obtained from MUA, in which multiple neurons are pooled together, thereby also leading to larger RFs. However, we cannot exclude the possibility that the relatively large annuli-and-wedge stimuli contribute to an overestimation of RF size. The precise influence of stimulus features on the resulting RF properties will need further detailed studies. Importantly, the estimation of RF location should remain mostly unaffected by this.

In conclusion, our work contributes to the rapidly growing field of marmoset monkey research. The concepts of less constrained paradigms, and the adaptation of stimuli to the ethological needs of a species might be transferred to other species and to other areas of research. Together, this will promote diversification of the animal model landscape (Hemberger et al., 2016; Keifer and Summers, 2016; Yartsev, 2017; Hale, 2019) and solidify the contribution of marmoset research.

\section{References}

Anton-Erxleben K, Carrasco M (2013) Attentional enhancement of spatial resolution: linking behavioural and neurophysiological evidence. Nat Rev Neurosci 14:188-200.

Belcher AM, Yen CC, Stepp H, Gu H, Lu H, Yang Y, Silva AC, Stein EA (2013) Large-scale brain networks in the awake, truly resting marmoset monkey. J Neurosci 33:16796-16804.

Benson NC, Jamison KW, Arcaro MJ, Vu AT, Glasser MF, Coalson TS, Van Essen DC, Yacoub E, Ugurbil K, Winawer J, Kay K (2018) The Human Connectome Project 7 Tesla retinotopy dataset: description and population receptive field analysis. J Vis 18:23.

Berger M, Calapai A, Stephan V, Niessing M, Burchardt L, Gail A, Treue S (2018) Standardized automated training of rhesus monkeys for neuroscience research in their housing environment. $J$ Neurophysiol 119:796-807.

Calapai A, Berger M, Niessing M, Heisig K, Brockhausen R, Treue S, Gail A (2017) A cage-based training, cognitive testing and enrichment system optimized for rhesus macaques in neuroscience research. Behav Res Methods 49:35-45.

Cardillo G (2012) Four parameters logistic regression - there and back again. Accessed October 11, 2020. Available from https://it. mathworks.com/matlabcentral/fileexchange/38122.

Carrasco M (2011) Visual attention: the past 25 years. Vision Res 51:1484-1525.

Chaplin TA, Yu H-HH, Rosa MGP (2013) Representation of the visual field in the primary visual area of the marmoset monkey: magnification factors, point-image size, and proportionality to retinal ganglion cell density. J Comp Neurol 521:1001-1019.

Citron MC, Emerson RC (1983) White noise analysis of cortical directional selectivity in cat. Brain Res 279:271-277.

Clopper CJ, Pearson ES (1934) The use of confidence or fiducial limits illustrated in the case of the binomial. Biometrika 26:404-413.

Courellis HS, NummelalD SU, Metke M, Diehl GW, Bussell R, CauwenberghsID G, MillerID CT (2019) Spatial encoding in primate hippocampus during free navigation. PLoS Biol 17:e3000546.

Ebina T, Obara K, Watakabe A, Masamizu Y, Terada S-I, Matoba R, Takaji M, Hatanaka N, Nambu A, Mizukami H, Yamamori T, Matsuzaki M (2019) Arm movements induced by noninvasive optogenetic stimulation of the motor cortex in the common marmoset. Proc Natl Acad Sci USA 116:22844-22850.

Ehinger BV, Groß K, Ibs I, König P (2019) A new comprehensive eyetracking test battery concurrently evaluating the Pupil Labs glasses and the EyeLink 1000. PeerJ 7:e7086.

Eliades SJ, Wang X (2008) Chronic multi-electrode neural recording in free-roaming monkeys. J Neurosci Methods 172:201-214.

Ferrari SF (2008) Predation risk and antipredator strategies. In: South American primates, pp 251-277. New York: Springer.

Fiorani M, Azzi JCB, Soares JGM, Gattass R (2014) Automatic mapping of visual cortex receptive fields: a fast and precise algorithm. J Neurosci Methods 221:112-126.

Gao L, Wang X (2019) Subthreshold activity underlying the diversity and selectivity of the primary auditory cortex studied by intracellular recordings in awake marmosets. Cereb Cortex 29:994-1005. 
Gao L, Kostlan K, Wang Y, Wang X (2016) Distinct subthreshold mechanisms underlying rate-coding principles in primate auditory cortex. Neuron 91:905-919.

Graham B, Judge SJ (1999) The effects of spectacle wear in infancy on eye growth and refractive error in the marmoset (Callithrix jacchus). Vision Res 39:189-206.

Guerrasio L, Quinet J, BüTtner U, Goffart L (2010) Fastigial oculomotor region and the control of foveation during fixation. $J$ Neurophysiol 103:1988-2001.

Hale ME (2019) Toward diversification of species models in neuroscience. Brain BehavEvol 93:166-168.

Harvey BM, Dumoulin SO (2011) The relationship between cortical magnification factor and population receptive field size in human visual cortex: constancies in cortical architecture. J Neurosci 31:1360413612.

Hassoumi A, Peysakhovich V, Hurter C (2019) Improving eye-tracking calibration accuracy using symbolic regression. PLoS One 14: e0213675.

Heide M, Haffner C, Murayama A, Kurotaki Y, Shinohara H, Okano H, Sasaki E, Huttner WB (2020) Human-specific ARHGAP11B increases size and folding of primate neocortex in the fetal marmoset. Science 369:546-550.

Hemberger M, Pammer L, Laurent G (2016) Comparative approaches to cortical microcircuits. CurrOpinNeurobiol 41:24-30.

Hessels RS, Kemner C, van den Boomen C, Hooge ITC (2016) The area-of-interest problem in eyetracking research: a noise-robust solution for face and sparse stimuli. Behav Res Methods 48:16941712.

Holmqvist K, Nyström M, Mulvey F (2012) Eye tracker data quality: what it is and how to measure it. In: Eye tracking research and applications symposium (ETRA). Santa Barbara, CA: Association for Computing Machinery.

Hubel DH, Wiesel TN (1962) Receptive fields, binocular interaction and functional architecture in the cat's visual cortex. J Physiol 160:106-154.

Hung CC, Yen CC, Ciuchta JL, Papoti D, Bock NA, Leopold DA, Silva AC (2015) Functional mapping of face-selective regions in the extrastriate visual cortex of the marmoset. $J$ Neurosci 35:1160-1172.

Johnston KD, Barker K, Schaeffer L, Schaeffer DJ, Everling S (2018) Methods for chair restraint and training of the common marmoset on oculomotor tasks. J Neurophysiol 119:1636-1646.

Jones JP, Stepnoski A, Palmer LA (1987) The two-dimensional spectral structure of simple receptive fields in cat striate cortex. J Neurophysiol 58:1212-1232.

Jun J, Mitelut C, Lai C, Gratiy S, Anastassiou C, Harris T (2017) Realtime spike sorting platform for high-density extracellular probes with ground-truth validation and drift correction. bioRxiv. doi: https://doi.org/10.1101/101030.

Kasprowski P, Hareôżlak K, Stasch M (2014) Guidelines for the eye tracker calibration using points of regard. Information Technologies in Biomedicine, Vol 4, pp 225-236. Cham: Springer.

Keifer J, Summers CH (2016) Putting the "biology" back into "neurobiology": the strength of diversity in animal model systems for neuroscience research. Front Syst Neurosci 10:69.

Kishi N, Sato K, Sasaki E, Okano H (2014) Common marmoset as a new model animal for neuroscience research and genome editing technology. Dev Growth Differ 56:53-62.

Knöll J, Pillow JW, Huk AC (2018) Lawful tracking of visual motion in humans, macaques, and marmosets in a naturalistic, continuous, and untrained behavioral context. Proc Natl Acad Sci USA 115: E10486-E10494.

Kondo T, Saito R, Otaka M, Yoshino-Saito K, Yamanaka A, Yamamori T, Watakabe A, Mizukami H, Schnitzer MJ, Tanaka KF, Ushiba J, Okano H (2018) Calcium transient dynamics of neural ensembles in the primary motor cortex of naturally behaving monkeys. Cell Rep 24:2191-2195.e4.

Koski SE, Burkart JM (2015) Common marmosets show social plasticity and group-level similarity in personality. Sci Rep 5:8878.
Krakauer JW, Ghazanfar AA, Gomez-Marin A, Maclver MA, Poeppel D (2017) Neuroscience needs behavior: correcting a reductionist bias. Neuron 93:480-490.

Liu C, Ye FQ, Yen CCC, Newman JD, Glen D, Leopold DA, Silva AC (2018) A digital 3D atlas of the marmoset brain based on multimodal MRI. Neuroimage 169:106-116.

Liu JV, Hirano Y, Nascimento GC, Stefanovic B, Leopold DA, Silva AC (2013) fMRI in the awake marmoset: somatosensory-evoked responses, functional connectivity, and comparison with propofol anesthesia. Neuroimage 78:186-195.

Macdougall M, Nummela SU, Coop S, Disney A, Mitchell JF, Miller CT (2016) Optogenetic manipulation of neural circuits in awake marmosets. J Neurophysiol 116:1286-1294.

Martinez LM, Wang Q, Reid RC, Pillai C, Alonso JM, Sommer FT, Hirsch JA (2005) Receptive field structure varies with layer in the primary visual cortex. Nat Neurosci 8:372-379.

Mathis A, Mamidanna P, Cury KM, Abe T, Murthy VN, Mathis MW, Bethge M (2018) DeepLabCut: markerless pose estimation of userdefined body parts with deep learning. Nat Neurosci 21:1281-1289.

Mehta P, Kreeger L, Wylie DC, Pattadkal JJ, Lusignan T, Davis MJ, Turi GF, Li WK, Whitmire MP, Chen Y, Kajs BL, Seidemann E, Priebe NJ, Losonczy A, Zemelman BV (2019) Functional access to neuron subclasses in rodent and primate forebrain. Cell Rep 26:2818-2832.e8.

Meyer AF, O'Keefe J, Poort J (2020) Two distinct types of eye-head coupling in freely moving mice. Curr Biol 30:2116-2130.

Miller CT, Freiwald WA, Leopold DA, Mitchell JF, Silva AC, Wang X (2016) Marmosets: a neuroscientific model of human social behavior. Neuron 90:219-233.

Mitchell JF, Leopold DA (2015) The marmoset monkey as a model for visual neuroscience. Neurosci Res 93:20-46.

Mitchell JF, Reynolds JH, Miller CT (2014) Active vision in marmosets: a model system for visual neuroscience. J Neurosci 34:11831194.

Mitchell JF, Priebe NJ, Miller CT (2015) Motion dependence of smooth pursuit eye movements in the marmoset. J Neurophysiol 113:39543960.

Mundinano IC, Fox DM, Kwan WC, Vidaurre D, Teo L, HommanLudiye J, Goodale MA, Leopold DA, Bourne JA (2018) Transient visual pathway critical for normal development of primate grasping behavior. Proc Natl Acad Sci USA 115:1364-1369.

Nath T, Mathis A, Chen AC, Patel A, Bethge M, Mathis MW (2019) Using DeepLabCut for 3D markerless pose estimation across species and behaviors. Nat Protoc 14:2152-2176.

Niehorster DC, Cornelissen THW, Holmqvist K, Hooge ITC, Hessels RS (2018) What to expect from your remote eye-tracker when participants are unrestrained. Behav Res Methods 50:213-227.

Niell CM, Stryker MP (2008) Highly selective receptive fields in mouse visual cortex. J Neurosci 28:7520-7536.

Nummela SU, Coop SH, Cloherty SL, Boisvert CJ, Leblanc M, Mitchell JF (2017) Psychophysical measurement of marmoset acuity and myopia. Dev Neurobiol 77:300-313.

Palmer J, Huk AC, Shadlen MN (2005) The effect of stimulus strength on the speed and accuracy of a perceptual decision. J Vis 5:376404.

Pandey S, Simhadri S, Zhou Y (2020) Rapid head movements in common marmoset monkeys. iScience 23:100837.

Park JE, Zhang XF, Choi S-H, Okahara J, Sasaki E, Silva AC (2016) Generation of transgenic marmosets expressing genetically encoded calcium indicators. Sci Rep 6:34931.

Paxinos G, Watson C, Petrides M, Rosa M, Tokuno H (2012) The marmoset brain in stereotaxic coordinates. San Diego: Elsevier.

Pfeuffer K, Vidal M, Turner J, Bulling A, Gellersen H (2013) Pursuit calibration: making gaze calibration less tedious and more flexible. UIST 2013. Proceedings of the 26th Annual ACM Symposium on User Interface Software and Technology, St. Andrews, Scotland, United Kingdom.

Poddar R, Kawai R, Ölveczky BP (2013) A fully automated highthroughput training system for rodents. PLoS One 8:e83171. 
Populin LC (2006) Monkey sound localization: head-restrained versus head-unrestrained orienting. J Neurosci 26:9820-9832.

Remington ED, Osmanski MS, Wang X (2012) An operant conditioning method for studying auditory behaviors in marmoset monkeys. PLoS One 7:e47895.

Ringach DL (2004) Mapping receptive fields in primary visual cortex. J Physiol 558:717-728.

Rolls ET, Aggelopoulos NC, Zheng F (2003) The receptive fields of inferior temporal cortex neurons in natural scenes. J Neurosci 232:339348.

Rosa MGP, Schmid LM (1995) Visual areas in the dorsal and medial extrastriate cortices of the marmoset. J Comp Neurol 359:272299.

Rosa MGP, Fritsches KA, Elston GN (1997) The second visual area in the marmoset monkey: visuotopicorganisation, magnification factors, architectonical boundaries, and modularity. J Comp Neurol 387:547-567.

Rosa MGP, Palmer SM, Gamberini M, Burman KJ, Yu HH, Reser DH, Bourne JA, Tweedale R, Galletti C (2009) Connections of the dorsomedial visual area: pathways for early integration of dorsal and ventral streams in extrastriate cortex. J Neurosci 29:4548-4563.

Roy S, Wang X (2012) Wireless multi-channel single unit recording in freely moving and vocalizing primates. I Neurosci Methods 203:28-40.

Sasaki E, Suemizu H, Shimada A, Hanazawa K, Oiwa R, Kamioka M, Tomioka I, Sotomaru Y, Hirakawa R, Eto T, Shiozawa S, Maeda T, Ito M, Ito R, Kito C, Yagihashi C, Kawai K, Miyoshi H, Tanioka Y, Tamaoki N, et al. (2009) Generation of transgenic non-human primates with germline transmission. Nature 459:523-527.

Sato K, Sasaguri H, Kumita W, Inoue T, Kurotaki Y, Nagata K, Mihira N, Sato K, Sakuma T, Yamamoto T, Tagami M, Manabe R, Ozaki K, Okazaki Y, Saido TC, Sasaki E(2020) A non-human primate model of familial Alzheimer's disease. bioRxiv2020.08.24.264259.

Schaeffer DJ, Gilbert KM, Hori Y, Gati JS, Menon RS, Everling S (2019) Integrated radiofrequency array and animal holder design for minimizing head motion during awake marmoset functional magnetic resonance imaging. Neuroimage 193:126-138.

Selvanayagam J, Johnston KD, Schaeffer DJ, Hayrynen LK, Everling $S$ (2019) Functional localization of the frontal eye fields in the common marmoset using microstimulation. J Neurosci 39:9197-9206.

Sereno MI, Dale AM, Reppas JB, Kwong KK, Belliveau JW, Brady TJ, Rosen BR, Tootell RBH (1995) Borders of multiple visual areas in humans revealed by functional magnetic resonance imaging. Science 268:889-893.

Servick K (2018) Why are U.S. neuroscientists clamoring for marmosets? Science 362:383-384.

Shen $\mathrm{H}$ (2013) Precision gene editing paves way for transgenic monkeys. Nature 503:14-15.

Solomon SG, Rosa MGP (2014) A simpler primate brain: the visual system of the marmoset monkey. Front Neural Circuits 8:96.

Sonkusare S, Breakspear M, Guo C (2019) Naturalistic stimuli in neuroscience: critically acclaimed. Trends Cogn Sci 23:699-714.
SR Research (2009) EyeLink 1000 user manual. Mississauga, Ontario, Canada: SR Research Ltd.

Stevenson MF, Poole TB (1976) An ethogram of the common marmoset (Calithrix jacchus jacchus): general behavioural repertoire. AnimBehav 24:428-451.

Tolias AS, Moore T, Smirnakis SM, Tehovnik EJ, Siapas AG, Schiller $\mathrm{PH}$ (2001) Eye movements modulate visual receptive fields of V4 neurons. Neuron 29:757-767.

Tomioka I, Ishibashi H, Minakawa EN, Motohashi HH, Takayama O, Saito Y, Popiel HA, Puentes S, Owari K, Nakatani T, Nogami N, Yamamoto K, Noguchi S, Yonekawa T, Tanaka Y, Fujita N, Suzuki H, Kikuchi H, Aizawa S, Nagano S, et al. (2017a) Transgenic monkey model of the polyglutamine diseases recapitulating progressive neurological symptoms. eNeuro 4:ENEURO.0250-16.2017.

Tomioka I, Nogami N, Nakatani T, Owari K, Fujita N, Motohashi H, Takayama O, Takae K, Nagai Y, Seki K (2017b) Generation of transgenic marmosets using a tetracyclin-inducible transgene expression system as a neurodegenerative disease model. Biol Reprod 97:772-780.

Tovee MJ, Rolls ET, Azzopardi P (1994) Translation invariance in the responses to faces of single neurons in the temporal visual cortical areas of the alert macaque. J Neurophysiol 72:1049-1060.

Umeda T, Koizumi M, Katakai Y, Saito R, Seki K (2019) Decoding of muscle activity from the sensorimotor cortex in freely behaving monkeys. Neuroimage 197:512-526.

Walker JD, Pirschel F, Gidmark N, MacLean JN, Hatsopoulos NG (2020) A platform for semiautomated voluntary training of common marmosets for behavioral neuroscience. J Neurophysiol 123:1420-1426.

Wallace DJ, Greenberg DS, Sawinski J, Rulla S, Notaro G, Kerr JND (2013) Rats maintain an overhead binocular field at the expense of constant fusion. Nature 498:65-69.

Wang S, Chandravadia N, Mamelak AN, Rutishauser U (2019) Simultaneous eye tracking and single-neuron recordings in human epilepsy patients. J Vis Exp. Advance online publication. Retrieved Jun 17, 2019. doi: 10.3791/59117.

Yamada H, Matsumoto N, Kimura M (2004) Tonically active neurons in the primate caudate nucleus and putamen differentially encode instructed motivational outcomes of action. J Neurosci 24:35003510.

Yang R, Zhang Z (2002) Model-based head pose tracking with stereovision. In: Proceedings of the 5th IEEE International Conference on Automatic Face Gesture Recognition, FGR 2002.

Yartsev MM (2017) The emperor's new wardrobe: rebalancing diversity of animal models in neuroscience research. Science 358:466469.

Young JW, Stricklen BM, Chadwell BA (2016) Effects of support diameter and compliance on common marmoset (Callithrix jacchus) gait kinematics. J Exp Biol 219:2659-2672.

Yu HH, Rowley DP, Price NSC, Rosa MGP, Zavitz E (2020) A twisted visual field map in the primate dorsomedial cortex predicted by topographic continuity. Sci Adv 6:eaaz8673. 